\title{
A particle size threshold governs diffusion and segregation of PAR-3 during cell polarization
}

\author{
Authors \\ Yiran Chang, Daniel J. Dickinson \\ Department of Molecular Biosciences, University of Texas at Austin
}

\section{Correspondence}

daniel.dickinson@austin.utexas.edu

\section{SUMMARY}

Regulation of subcellular components' localization and motion is a critical theme in cell biology. Cells use the actomyosin cortex to regulate protein distribution on the plasma membrane, but the interplay between membrane binding, cortical movements and protein distribution remains poorly understood. In a polarizing one-cell stage Caenorhabditis elegans embryo, actomyosin flows transport PAR protein complexes into an anterior cortical domain to establish the anterior-posterior axis of the animal. Oligomerization of a key scaffold protein, PAR-3, is required for aPAR cortical localization and segregation. Although PAR-3 oligomerization is essential for polarization, it remains unclear how oligomer size contributes to aPAR segregation because PAR-3 oligomers are a heterogeneous population of many different sizes. To address this question, we engineered PAR-3 to defined sizes. We report that PAR-3 trimers are necessary and sufficient for PAR-3 function during polarization and later embryo development, while larger PAR-3 clusters are dispensable. Quantitative analysis of PAR-3 diffusion showed that PAR-3 clusters larger than a trimer are transported by viscous forces without being physically captured by the actomyosin cortex. Our study provides a quantitative model for size-dependent protein transportation of membrane proteins by cortical flow. 


\section{INTRODUCTION}

Subcellular components must be properly localized for normal cellular function. Cells have evolved a variety of mechanisms to position subcellular components as large as organelles and as small as single mRNA and protein molecules. The actomyosin cytoskeleton is actively involved in many of these mechanisms. For instance, myosin motor proteins transport large cargos, such as vesicles, by 'walking' on actin filaments (Mehta, 2001), and chromosome congression in starfish oocytes is accomplished by chromosome trapping within the contracting filamentous actin meshwork (Lénárt et al., 2005). Bulk cytoplasmic and cortical flows, triggered by non-muscle myosin dependent cytoskeleton contraction, are also responsible for the advective transport of a variety of subcellular components in a wide range of organisms, ranging from the circulation of chloroplasts in Elodea leaf cells (Allen and Allen, 1978) to the segregation of PAR proteins in Caenorhabditis elegans embryos (Lang and Munro, 2017). However, it has remained unclear how cortical flows can overcome the random motion caused by Brownian diffusion in order to transport macromolecular complexes over large distances.

The C.elegans zygote has been widely used to study protein segregation by actomyosin flows due to its relatively large size, optical transparency and the genetic tools that are available. During the first cell cycle of $C$. elegans embryonic development, distinct groups of PAR polarity proteins are segregated to anterior and posterior poles of the cell cortex, leading to asymmetric cell division which is essential for subsequent development. The anterior PAR complex (aPAR) consists of the central oligomeric scaffold protein PAR-3, along with atypical protein kinase C (aPKC/PKC-3) and its cofactor PAR-6. These proteins are distributed uniformly throughout the entire embryo cortex before polarization starts (Cuenca et al., 2003; Lang and Munro, 2017). Upon symmetry breaking triggered by sperm entry, a gradient in actomyosin contractility along the anterior-posterior axis is introduced, and anterior directed cortical and cytoplasmic flows are triggered as a result (Mayer et al., 2010; Munro et al., 2004). aPAR complexes are carried toward the anterior pole by this cortical flow and become enriched at the anterior cell cortex. Once localized, aPKC phosphorylates substrates essential for polarized cell behavior (Hong, 2018; Lang and Munro, 2017).

Multiple studies using different methods have shown that cortical flow is responsible for aPAR segregation in C. elegans zygotes. Munro et al. and Shelton et al. demonstrated that cortical flow is required for aPARs segregation by eliminating cortical flow with myosin light chain (MLC-4) RNAi, which results in PAR polarity defect (Munro et al., 2004; Shelton et al., 1999). Using modeling approaches, Grill and colleagues have shown that advective flow can be sufficient to explain PAR protein partitioning in silico (Goehring et al., 2011; Gross et al., 2019). Finally, direct induction of cytoplasmic flow by localized laser-induced heating was found to be sufficient to displace the aPAR cortical domain in vivo (Mittasch et al., 2018).

However, these studies have not addressed how cortical flows physically transport PAR proteins. The cell cortex consists of actin and myosin filaments as well as surrounding protein and water molecules, but models generally treat the entire cortex as a film of active fluid (Goehring et al., 2011; Gross et al., 2019; Mayer et al., 2010). Therefore, it remains unclear 
whether cortical PAR proteins are pushed along the membrane by physical association with actin filaments or, instead, transported through viscous forces generated by cytoplasmic flow.

Clustering of aPAR, which occurs due to PAR-3 oligomerization, is critical for aPAR localization across a range of organisms and cell types (Benton and Johnston, 2003; Dickinson et al., 2017; Mizuno et al., 2003; Rodriguez et al., 2017; Sailer et al., 2015; Wang et al., 2017a). Using CRISPR-induced targeted mutagenesis and live imaging of C.elegans embryos, we previously showed that PAR-3 clusters of different oligomer sizes have distinct responses to cortical flow, while mutants in which PAR-3 is strictly monomeric have severe polarity defects and are unable to segregate aPAR (Dickinson et al., 2017). These results hinted at a model in which a large size enables PAR-3 clusters to be transported by cortical flow for proper polarity establishment. However, since PAR-3 oligomers have a wide range of sizes in vivo (Dickinson et al., 2017; Lang and Munro, 2017; Wang et al., 2017b), it remains unclear how oligomer size contributes to aPAR segregation.

Here, we address this question by performing a quantitative analysis of oligomerization-dependent protein segregation by actomyosin cortical flow. By dissecting out the heterogeneous PAR-3 population through engineered PAR-3 variants that form oligomers of defined sizes, we reveal that PAR-3 clusters diffuse freely through the actomyosin cortex, and segregate to the anterior cortical domain as a result of viscous friction rather than direct association with actomyosin. These results provide fundamental insights into the mechanisms of polarization and of protein transport by cortical flows.

\section{RESULTS}

\section{Engineered PAR-3 trimers are sufficient for aPAR segregation and normal development}

Wild-type PAR-3 exists in vivo as a heterogeneous population of oligomers of different sizes, ranging from monomers to >15-mers (Dickinson et al., 2017). In previous work, we disrupted the PAR-3 oligomerization domain by introducing charge-reversal mutations at three key positions in the endogenous par-3 gene using CRISPR. The resulting PAR-3(RRKEEE) monomeric mutant protein (here referred to as PAR- $3^{*}$ ) does not localize stably to the cell membrane or segregate to the anterior domain (Dickinson et al., 2017) (see Figures 1B-C). In the same study, we also showed that the brightest $25 \%$ of PAR-3 clusters segregated efficiently on the cell cortex in living embryos, while the dimmest $25 \%$ moved shorter distances in an apparently diffusive manner. Therefore, large PAR-3 clusters, formed by oligomerization, were presumed to play an important functional role in polarity establishment. However, the exact relationship between PAR-3 cluster size and segregation remained unclear. To dissect the behavior of the heterogenous PAR-3 population and to determine how differently-sized PAR-3 clusters are transported by cortical flow, we engineered PAR-3 oligomers of controlled sizes.

We tested a handful of protein domains that have been reported to autonomously organize into oligomers of defined sizes in vitro (Figure S1A-B) (Bolten et al., 2016; Boudko et al., 2009; 
Büttner et al., 2012; Chik et al., 2019; Drulyte et al., 2019; Huang et al., 2014; Lee et al., 1968; Li et al., 2019; Luo et al., 2001; Parsons et al., 2002; Santiago-Frangos et al., 2019; Sun et al., 2014; Thomson et al., 2014; Veesler et al., 2010). Each oligomerization domain was tagged with a fluorescent protein and expressed in C.elegans, and the size of each oligomer protein complex from the transgenic zygotes is examined using single-molecule pull-down (Sc-SiMPull) followed by photobleaching step counting (Dickinson et al., 2017). We identified dimer, trimer, tetramer and hexamer protein domains that formed oligomers of the expected sizes in vivo (Figure S1A-C).

To generate PAR-3 oligomers of defined sizes, we adopted a strategy in which PAR-3 is linked to one of these "extra oligomerization domains" (EODs) via a nanobody. We generated transgenic constructs consisting of a nanobody that binds to GFP/YFP (Wang et al., 2017a), a fluorescent BFP or HaloTag and an EOD (Figure 1A iii). We verified that the nanobody successfully bound to GFP/YFP in C. elegans lysates in vitro (Figure S1D) and in live embryos in vivo (see below). To generate engineered strains with PAR-3 oligomers of controlled sizes, we crossed each $n A b:: E O D$ construct to a strain carrying an endogenous YFP-tagged PAR-3 monomer allele (YFP::PAR-3*(RRKEEE)) (Figure 1A).

Strikingly, our 3mer, 4 mer and 6 mer nAb::EOD constructs fully rescued the polarity defects in the parental YFP::PAR $-3^{*}$ monomeric strain. Figure 1B illustrates this rescue using one of the 6 mer constructs (HOTag3) as an example. Neither the 6mer(HOTag3) EOD construct nor YFP::PAR-3* monomer by itself localized to the cortex or adopted a polarized distribution, but when the 6mer(HOTag3) EOD construct and YFP::PAR-3* were present together, the two proteins colocalized in clusters that segregated to the anterior half of the zygote (Figures 1B). The engineered $3 \mathrm{mer}(3 \mathrm{HON})$, another $6 \mathrm{mer}(6 \mathrm{GW} 3)$ and two different $4 \mathrm{mers}(\mathrm{AraD}$ and Yrb1) also formed clusters that segregated to the anterior (Figure 1C-D and Movie S1).

A negative control strain without any EOD, nAb::Halo;YFP::PAR-3* ${ }^{*}$ closely resembled the YFP::PAR-3* mutant: we observed only very weak membrane binding, with only a few clusters visiting the membrane for a very short amount of time and without forming a polarized anterior domain (Figure 1C and Movie S1). The engineered 2mer (6HNL) PAR-3 exhibited an intermediate phenotype: PAR-3 dimers localized to the membrane with shorter membrane binding lifetime (Figure 4C and Movie S1). A minority of 2 mer embryos (4/14) showed some ability to polarize, but most embryos (10/14) were not polarized effectively (Figure 1C-D and $\mathrm{S} 1 \mathrm{E})$. These data indicate that a threshold size of three PAR-3 molecules per cluster is necessary and sufficient for robust polarity establishment in the $C$. elegans zygote.

On the phenotypic level, strains carrying the monomeric PAR $-3^{*}$ mutation exhibit partially penetrant embryonic lethality and adult sterility (Dickinson et al., 2017). Strikingly, these phenotypes were almost fully rescued by the 3mer, 4 mer or 6 mer EOD::nAb constructs, and slightly rescued by the 2 mer construct (Figure $1 \mathrm{E}-\mathrm{F}$ ). We did observe some mild phenotypes in individual engineered strains: the PAR $-3^{*} ; 6 \operatorname{mer}(\mathrm{HOTag} 3)$ strain had slow larval growth and maturation, and a few embryos $(<10 \%)$ of the PAR-3*;4mer(AraD) strain exhibited cytokinesis defects. These phenotypes have no obvious relationship to cell polarity, and we presume that they are artifacts of expressing these specific EODs. Nevertheless, the recovery of PAR-3 
bioRxiv preprint doi: https://doi.org/10.1101/2021.06.07.447386; this version posted June 7, 2021. The copyright holder for this preprint (which was not certified by peer review) is the author/funder, who has granted bioRxiv a license to display the preprint in perpetuity. It is made available under aCC-BY-NC-ND 4.0 International license.

localization, zygote polarity, embryonic lethality and adult sterility indicate that oligomers as small as trimers are sufficient for PAR-3 function in $C$. elegans. 
bioRxiv preprint doi: https://doi.org/10.1101/2021.06.07.447386; this version posted June 7, 2021. The copyright holder for this preprint (which was not certified by peer review) is the author/funder who has granted bioRxiv a license to display the preprint in perpetuity. It is made available underaed-BY-NC-ND 4.0 International license.

A
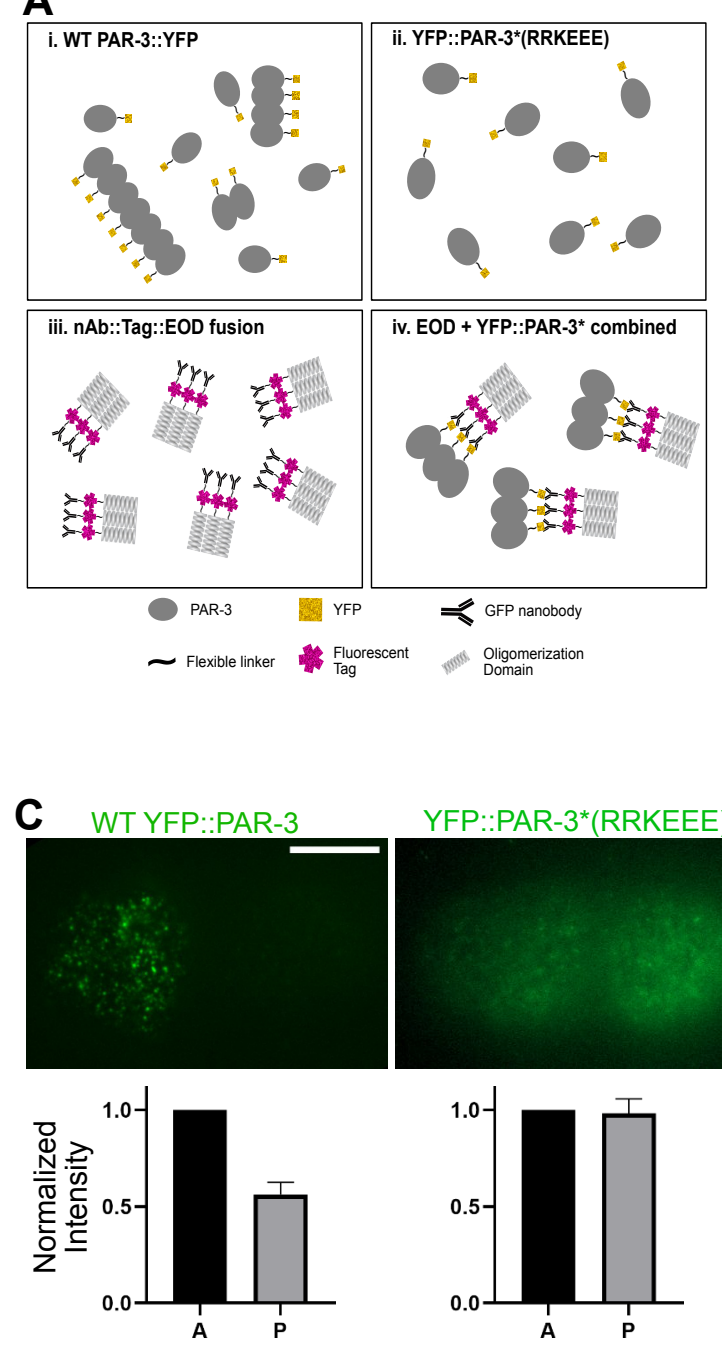

$\mathrm{nAb}:: \mathrm{BFP}:: 3 \mathrm{mer}(3 \mathrm{HON})$;
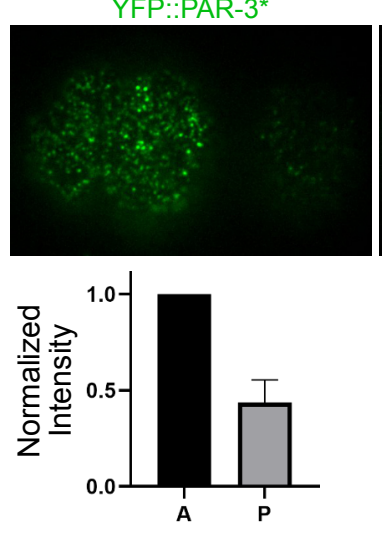

D

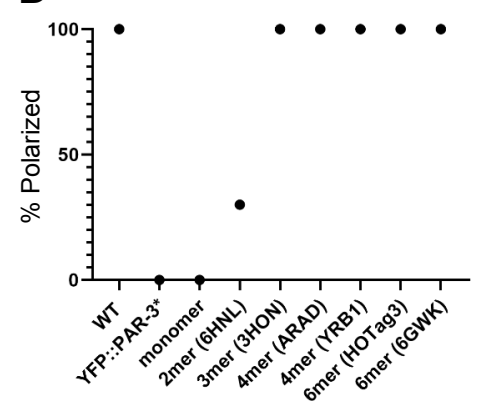

E

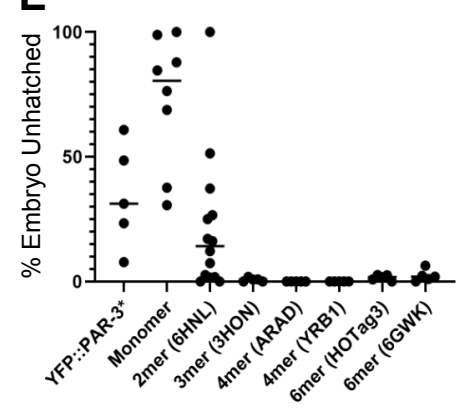

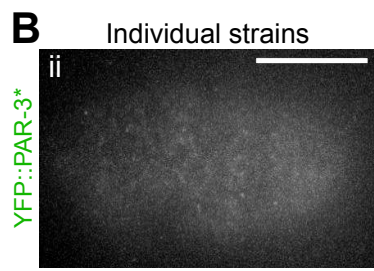
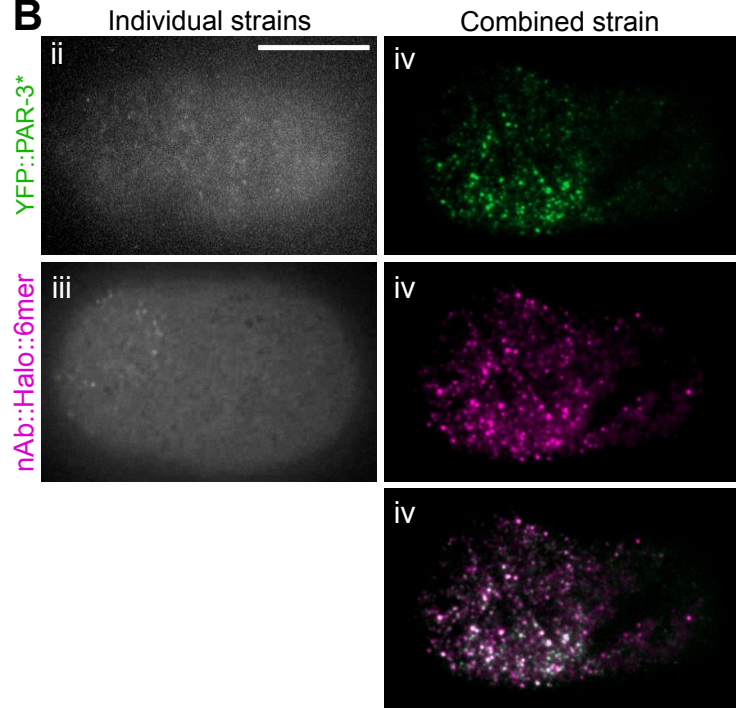

nAb::BFP::2mer(6HNL); YFP::PAR-3*
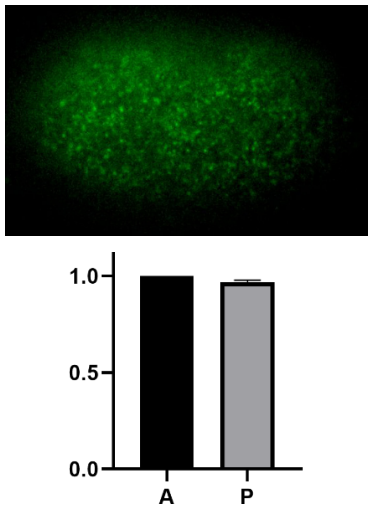

nAb::BFP::6mer(6GWK);

nAb::BFP::4mer(Yrb1);
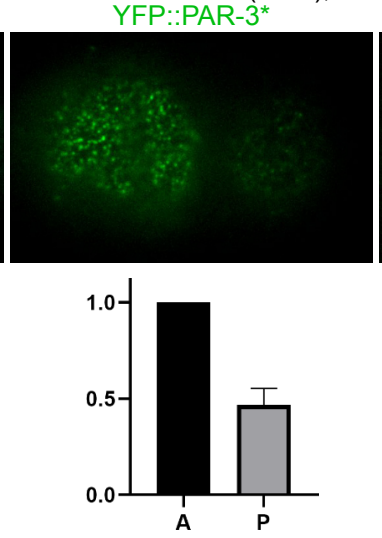
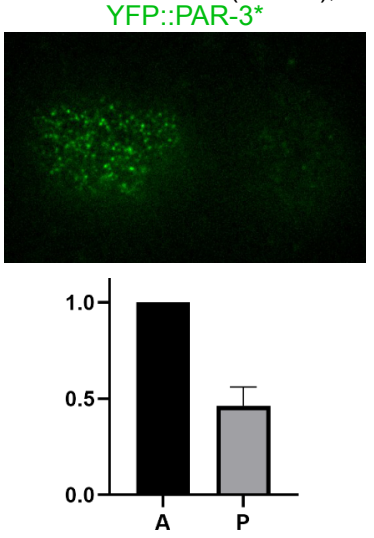

$F$

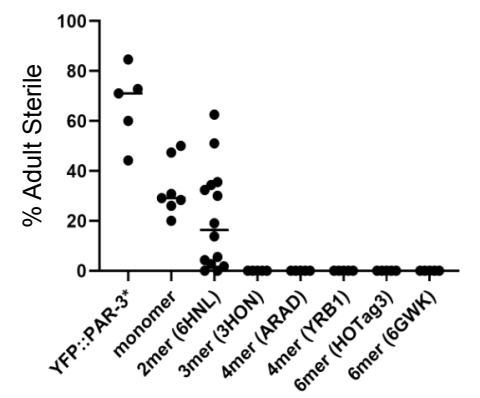


Figure 1. Engineered PAR-3 trimers are sufficient for aPAR segregation and normal development

(A) Illustration of our strategy to engineer PAR-3 to specific sizes. i. During polarity establishment, PAR-3 clusters exist as a heterogeneous population of various sizes. ii. PAR-3 monomers in a mutant embryo in which the PAR-3 oligomerization domain is disrupted. iii. Transgenic EOD constructs contain the EOD (3mer is shown as an example), a fluorescent label and a GFP-binidng nanobody. iv. PAR-3 of controlled oligomer size was generated by combining YFP::PAR-3*(RRKEEE) and EOD constructs.

(B) Detailed illustration of the rescue experiment result, hexamer(HOTag3) was shown as an example.

Left panels: (Top) TIRF imaging of embryo from YFP.:PAR-3* monomeric mutant strain after polarization stage, corresponding to ii in panel A. (Bottom) TIRF imaging of embryos from GFPnanobody::HaloTag::6mer(HOTag3) construct strain after polarization stage, corresponding to iii in panel $A$.

Right panels: TIFR imaging of polarized zygote from YFP::PAR-3;nAb::HaloTag::6mer(HOTag3) strain, corresponding to iv in panel A (Top) YFP.:PAR-3 channel. (Middle)

nb::Halo::6mer(HOTag3) channel. (Bottom) composite image with both YFP and Halo signal.

Scale bar: $10 \mu \mathrm{m}$.

(C) Upper panels: Live images of cortical YFP::PAR-3* in the indicated EOD strains. Scale bar:

$10 \mu \mathrm{m}$. Lower panels: Quantification of fluorescence intensity in posterior area to anterior area.

(D) Percentage of EOD strains that have effective PAR-3 polarization. $n=10$.

(E) Embryo lethality of each EOD strain. "Monomer" is a control construct comprising the nanobody and fluorescent tag but no EOD.

(F) Adult sterility of each EOD strain. "Monomer" is a control construct comprising the nanobody and fluorescent tag but no EOD 


\section{PAR-3 dimers exhibit brownian diffusion while larger oligomers undergo directed motion}

We were intrigued by the observation that an engineered PAR-3 trimer was sufficient to enable cell polarization, but a dimer was not. To attempt to explain why cortical flow is effective on trimers but not on dimers, and to better define the movement of PAR-3 on the cortex, we analyzed the diffusive behavior of individual engineered PAR-3 clusters. We imaged one-cell embryos of each EOD strain during polarity establishment using TIRF microscopy at a high frame rate (20 frames/second) to visualize the diffusion of clusters on the cortex and then computationally segmented and tracked individual particles (Figure 2A). To characterize and compare the motion of different-sized clusters, we performed a Mean Squared Displacement (MSD) analysis. The diffusion of particles on a 2D surface, such as the cell membrane, can be described as:

$$
M S D=<x(t)^{2}>=4 D t^{\alpha}
$$

Where $D$ is the diffusion coefficient and $\alpha$ is the anomalous diffusion parameter. An $\alpha<1$ indicates sub-diffusion where the movement of a particle is confined. $\alpha=1$ describes normal Brownian diffusion. $\alpha>1$ represents super-diffusion, which indicates directed movement of particles (Figure 2B). Therefore, the anomalous parameter provides direct information about the type of motion a particle is experiencing.

To measure the anomalous diffusion parameter $\alpha$, we fit the MSD curves (Figure 2C) for each individual particle track to the equation $y=k x^{\alpha}$, where the fitting parameter ' $k$ ' corresponds to $4^{*} \mathrm{D}$ and the fitting parameter ' $\alpha$ ' corresponds to the anomalous parameter. We repeated this experiment and analysis with $3 \operatorname{mer}(3 \mathrm{HON}), 4 \mathrm{mers}(\mathrm{Yrb} 1)$, and $6 \operatorname{mer}(6 \mathrm{GW} 3)$ EOD strains and calculated the anomalous parameter and diffusion coefficient for every single track. The 4 mer(AraD) and 6mer(HOTag3) EOD strains were not included in this analysis due to the mild, non-polarity related phenotypes noted above.

We observed a threshold in the anomalous parameter between engineered dimer and other larger engineered oligomers. The mean anomalous parameter was closer to $\alpha=2$ for $3 \mathrm{mer}, 4 \mathrm{mer}$ and 6mer EOD strains, indicating that PAR-3 oligomers engineered to contain 3 or more monomers underwent directed motion due to cortical flow (Figure 2D). In contrast, dimers moved in a much less directed fashion, indicated by the fact that the anomalous parameter $\alpha$ was only slightly greater than 1 (Mean $\pm 95 \% \mathrm{Cl}$ of $1.34 \pm 0.13$; Figure $2 \mathrm{D}$, left). There was no measurable difference in the $\alpha$ value between 3mer, 4 mer and 6 mer EOD strains, consistent with the observation that all three EOD constructs rescued normal polarization and embryo viability (Figure 1). The observed difference between the dimers and larger oligomers is consistent with the polarity and embryo lethality phenotypes we observed in the 2 mer strain. We conclude that during polarity establishment, engineered 3mers, 4mers and 6mers of PAR-3 undergo efficient directed motion due to cortical flow, while 2 mers move in a more diffusive fashion and are only weakly affected by cortical flow. 
bioRxiv preprint doi: https://doi.org/10.1101/2021.06.07.447386; this version posted June 7, 2021. The copyright holder for this preprint (which was not certified by peer review) is the author/funder who has granted bioRxiv a license to display the preprint in perpetuity. It is made available under aCC-BY-NC-ND 4.0 International license.

A

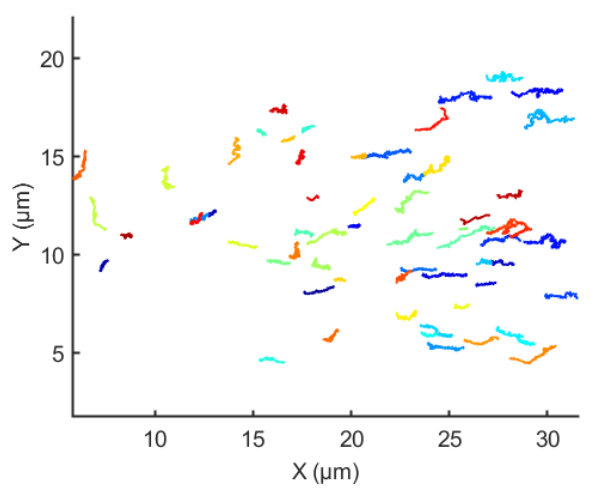

C

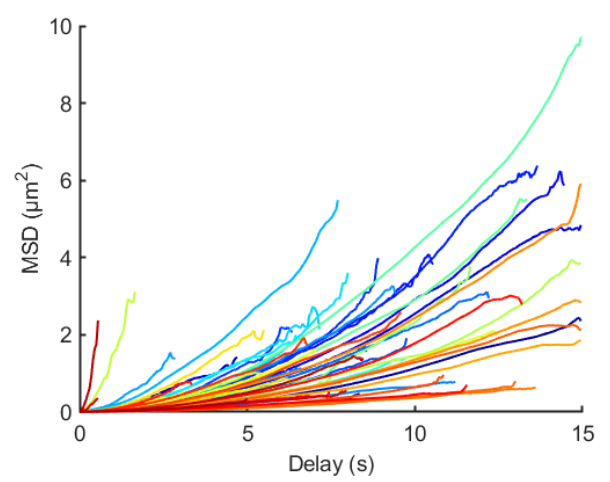

E

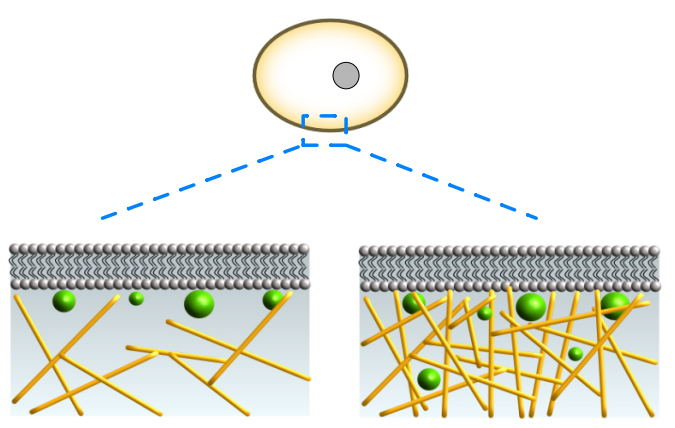

F
B

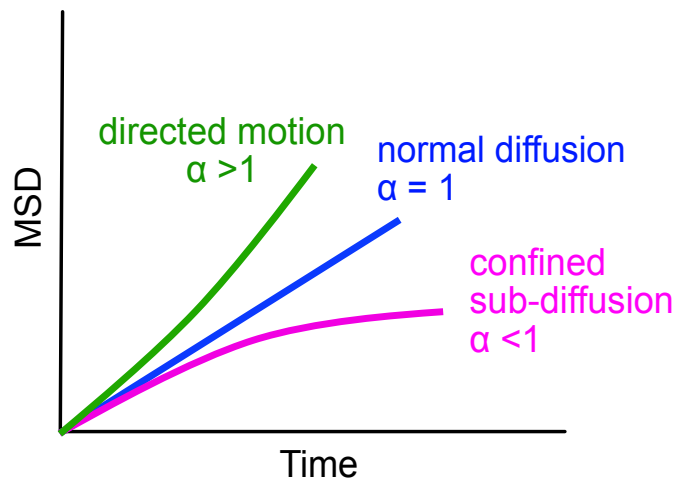

D
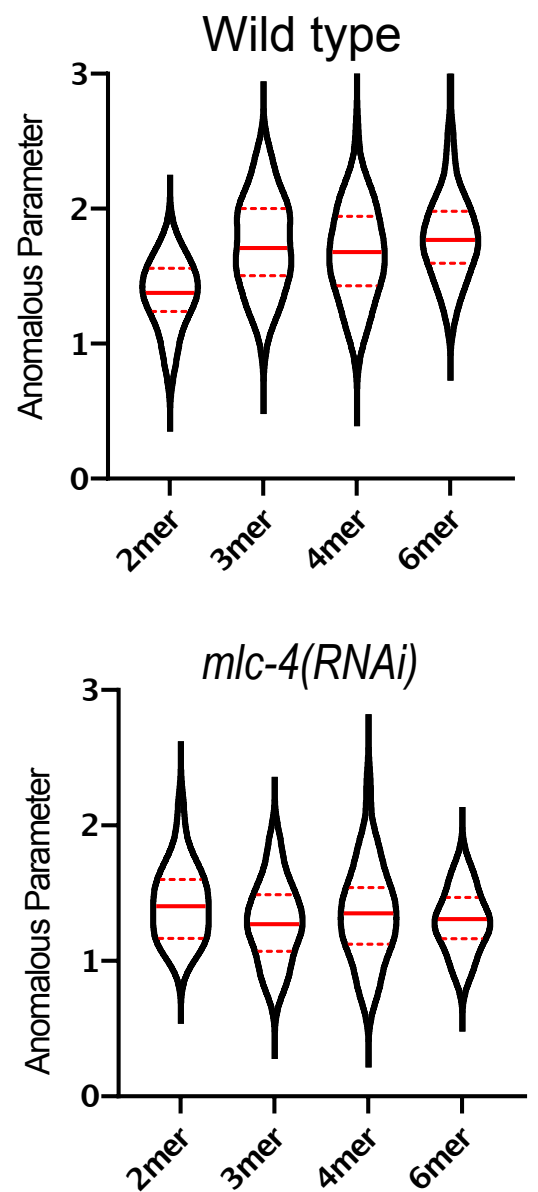
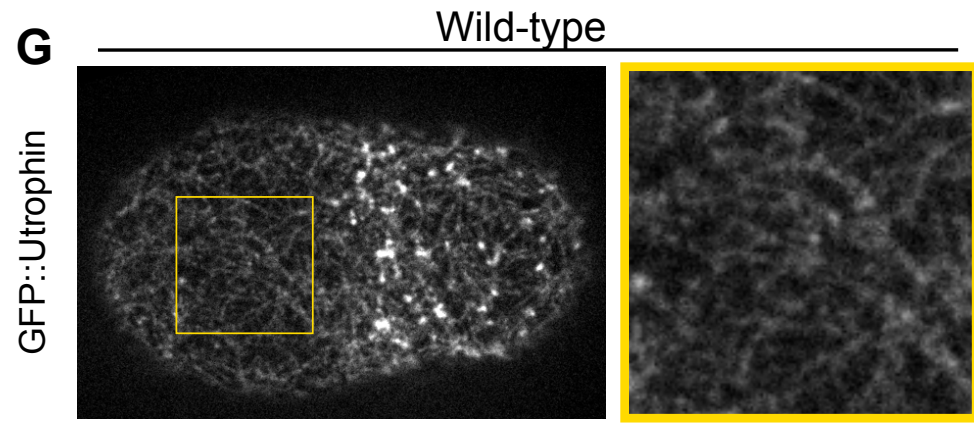

mlc-4(RNAi)
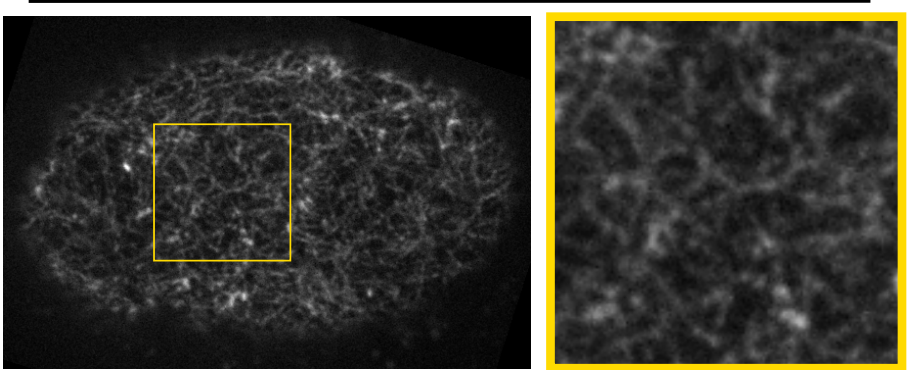


\section{Figure 2. PAR-3 dimers exhibit brownian diffusion while larger oligomers undergo} directed motion

(A) Tracks of engineered PAR-3 trimers imaged at the cortex during polarity establishment. Anterior to the left. Different colors are only used for better visualization. $t=15$ seconds.

(B) The interpretation of anomalous parameters.

(C) MSD plots of engineered trimers. Each curve describes the displacement of a single PAR-3 cluster as shown in (A).

(D) Measurements of the anomalous diffusion parameter, $\alpha$, in engineered PAR-3 strains. Solid lines indicate means. Dashed lines indicate upper and lower quartiles. Data points pooled from 3 embryos.

(E) Illustration of two alternative hypothesized models of PAR-3 cortical motion and interaction with actomyosin. Left: PAR-3 diffuses freely across the actomyosin cortex meshwork regardless of oligomer size. Right: PAR-3 is trapped in the pores of the actomyosin cortex once it reaches a certain size.

(F) Measurements of the anomalous diffusion parameter, $\alpha$, in engineered PAR-3 strains with MLC-4 RNAi. Solid lines indicate means. Dashed lines indicate upper and lower quartiles. Data points pooled from 3 embryos.

(G) Super-resolution iSIM images of GFP::UTRO zygotes at the pronuclei meeting stage, with or without $m / c-4$ RNAi. The structure of the actin network is intact under MLC-4 RNAi. Scale bar = $10 \mu \mathrm{m}$. Yellow box: region enlarged to the right of each image. 


\section{PAR-3 clusters and cortical actin move in tandem but are not physically associated}

To address the physical explanation for the difference in diffusive behavior between PAR-3 dimers and larger clusters, we considered the relationship between PAR-3 and the actomyosin cortex. We sought to test two competing hypotheses (Figure 2E). First, the dominant physical force for PAR-3 cluster segregation might be a viscous force, generated from the friction between the PAR-3 clusters and the flowing membrane or cortical cytosol. Alternatively, the larger PAR-3 clusters might be physically trapped in the actomyosin meshwork and pushed forward due to contacts with the actomyosin cortex. This second hypothesis predicts that there will be a threshold in size, related to the effective pore size of the actomyosin meshwork, that allows PAR-3 clusters to be trapped in the actomyosin cortex and transported to the anterior domain along with the anterior directed cortex contraction.

To distinguish between these two hypotheses, we sought to directly test whether the larger PAR-3 oligomers are confined within the actomyosin meshwork of the cortex. We repeated the imaging and MSD analysis in our EOD strains after eliminating cortical flow by depleting myosin light chain ( $\mathrm{m} / \mathrm{c}-4 \mathrm{RNAi}$ ), which allows us to observe the behavior of PAR-3 clusters in the absence of cortical flow. If larger PAR-3 oligomers were confined within the actomyosin meshwork, then the anomalous diffusion parameter should shift from $\alpha>1$ (directed motion) to $\alpha<1$ (confined diffusion) when cortical flow is eliminated. We found that when cortical flow was absent, the anomalous parameters for $3 \mathrm{mer}$, $4 \mathrm{mer}$ and 6 mer were significantly reduced, but still slightly greater than 1 , while the anomalous parameter for 2 mer remained unchanged (Figure $2 \mathrm{~F}$ ). These results suggested that PAR-3 clusters diffuse freely on the membrane and are not confined by the actin cortex. Importantly, m/c-4 RNAi eliminated cortical flow but did not grossly disrupt the organization of the actin cortex (Figure 2G), suggesting that the absence of a confined diffusion behavior of PAR-3 oligomers under mlc-4 RNAi treatment (Figure 2F) is not likely to be caused by a change in the actin network architecture.

As a second test, we sought to directly visualize the relationship between PAR-3 and F-actin. Although the flow of actomyosin cortex and PAR-3 clusters are coupled, there is no known binding interaction between PAR-3 and F-actin. The relationship between PAR-3 and actomyosin cortex has been directly visualized by observing labeled myosin II (NMY-2) (Movie S2) (Dickinson et al., 2017), but F-actin and PAR-3 have not been visualized together in living embryos to our knowledge. Therefore, we constructed a strain carrying endogenously tagged mScarlet::PAR-3 and a transgenic GFP::utrophin reporter that binds to F-actin (Tse et al., 2012) by endogenously tagging PAR-3 with mScarlet-I in a GFP::utrophin background. We first imaged zygotes from this strain at high speed (3seconds/frame) using TIRF microscopy (Figure S3A and Movie S3). In agreement with previous studies, live fluorescence imaging showed that the movement of the actin cortex and of PAR-3 are tightly coupled (Goehring et al., 2011; Munro et al., 2004) (Figure 3A-B and Movie S3).

To quantify the correlation between PAR-3 segregation and actin flow, we selected 2 time points during the polarity establishment: one when the cortex was actively contracting towards the anterior pole and one when the cortex was temporarily oscillating and moving towards the future dorsal side. We quantified the movements using particle image velocimetry (PIV) and compared 
the vector fields for PAR-3 and F-actin using Pearson's correlation (Figure S3B-C). As expected, we observed a positive correlation between PAR-3 movement and F-actin movement (Pearson's correlation $\mathrm{R}=0.70$ for $\Delta \mathrm{t} 1, \mathrm{R}=0.59$ for $\Delta \mathrm{t} 2$ ), indicating that PAR-3 and the actomyosin cortex move in tandem. Nevertheless, PAR-3 and actin did not colocalize or overlap with each other in TIRF images (Pearson's correlation $R=0.067 \pm 0.010$; Figure 3A-B).

Although we did not observe an obvious association between PAR-3 and actin in TIRF images, it remained possible that contacts between actin and PAR-3 might have been overlooked due to the density of the F-actin network and the diffraction-limited resolution of our TIRF images.

Therefore, to better visualize the interaction between PAR-3 and the actin network, we performed super-resolution imaging of the cortex using Instant Structured Illumination (iSIM) (Figure 3C). We found that the PAR-3 clusters appeared as point sources even in super-resolution images, suggesting that even the largest clusters are smaller than the $\sim 170 \mathrm{~nm}$ resolution of iSIM imaging (Figure 3D). In contrast, individual actin branches and pores in the actomyosin cortex were clearly visible (Figure 3D). These observations reveal important information about the scale of sizes: PAR-3 clusters are much smaller than the gaps in the actomyosin cortex. Furthermore, PAR-3 clusters were frequently found within the pores of the cortex, not associated with individual filaments (Figure 3D).

Together, these data rule out the hypothesis that the directed movement of larger PAR-3 oligomers is due to physical trapping within the actomyosin meshwork, and instead indicate that PAR-3 clusters, regardless of their size, can diffuse freely on the membrane without being confined by the actomyosin cortex. We infer that PAR-3 segregation is likely driven by viscous forces, generated by the flowing cortical cytosol and/or membrane. 
bioRxiv preprint doi: https://doi.org/10.1101/2021.06.07.447386; this version posted June 7, 2021. The copyright holder for this preprint (which

was not certified by peer review) is the author/funder, who has granted bioRxiv a license to display the preprint in perpetuity. It is made available under aCC-BY-NC-ND 4.0 International license.

A

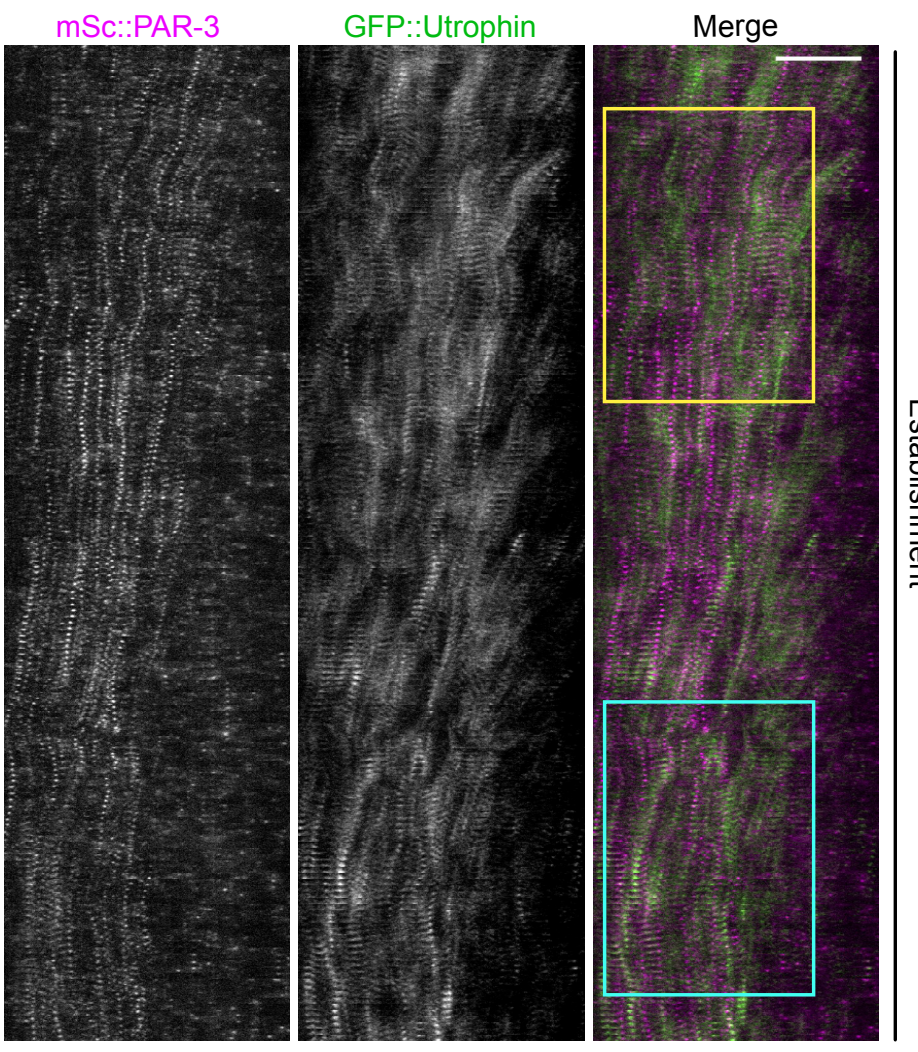

B

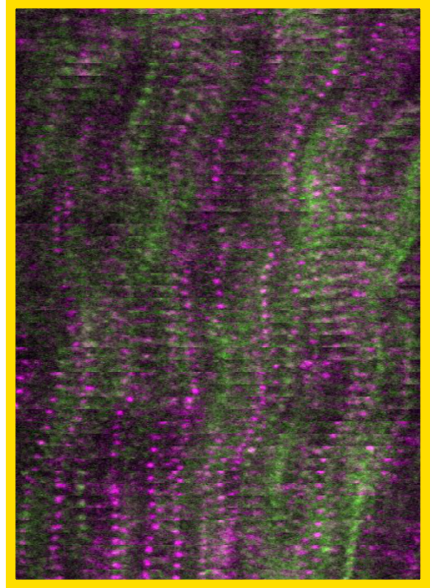

GFP::Utrophin
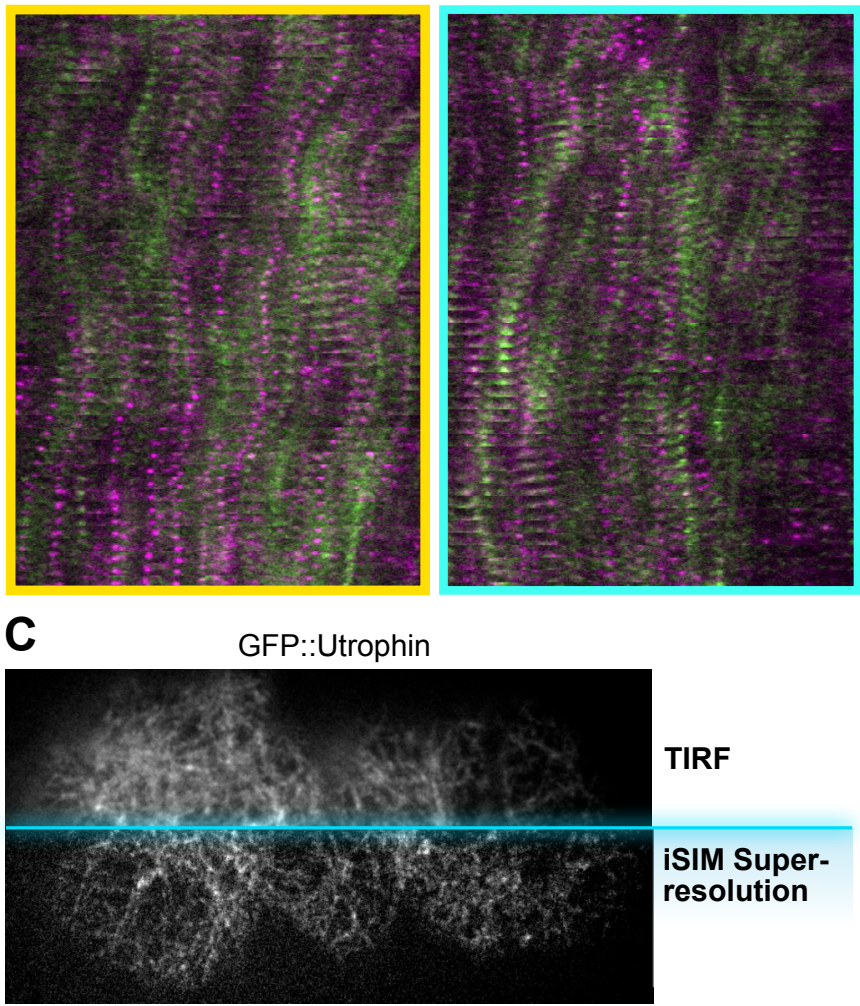

C

TIRF

iSIM Superresolution
D

mSc:::PAR-3
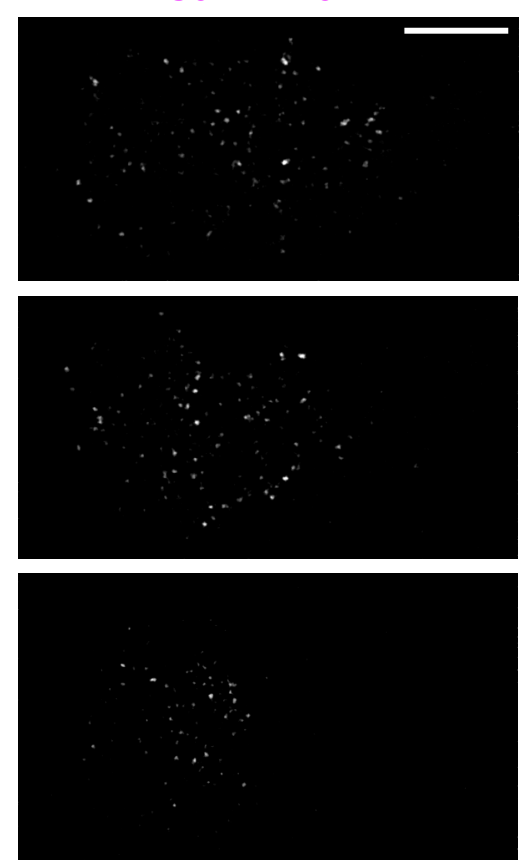

GFP::Utrophin
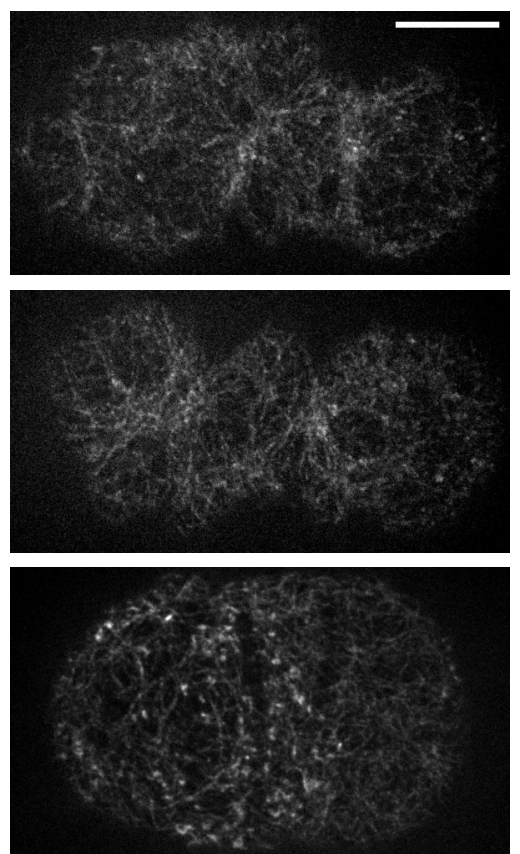

Merge
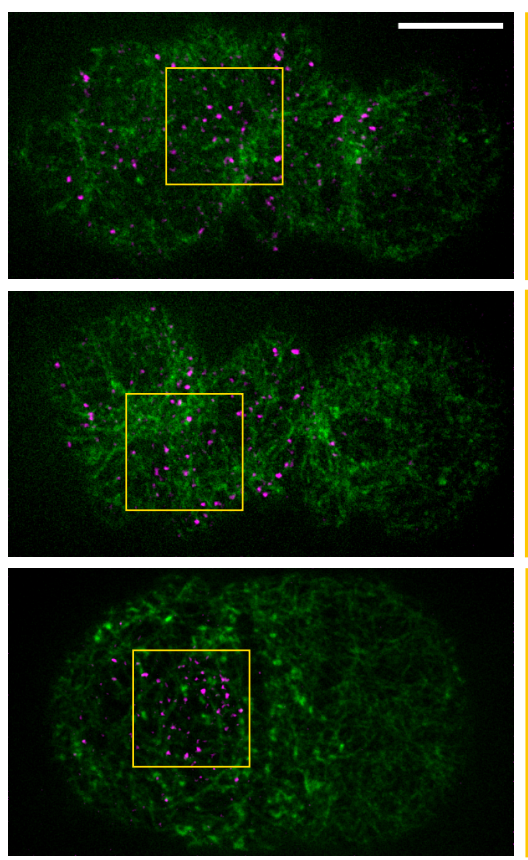

Enlarged

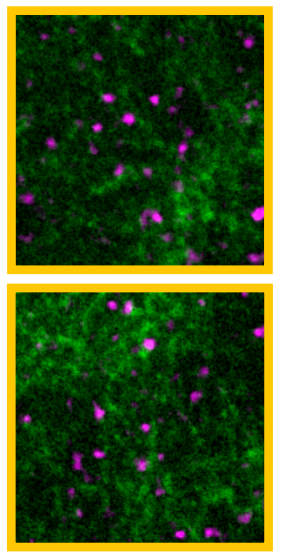

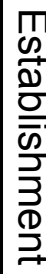

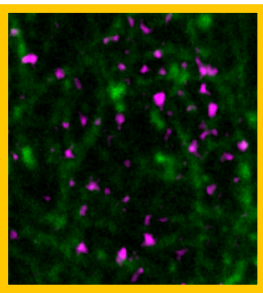


Figure 3. Super-resolution imaging reveals that PAR-3 moves in tandem with the actomyosin cortex despite not being physically associated with actin

(A) Kymograph of cortical mSc::PAR-3 and Utrophin::GFP during the first cell cycle. Imaged using TIRF at 3 seconds/frame. Anterior to the left. Scale bar $=10 \mu \mathrm{m}$. Yellow/Blue boxes: region enlarged in (B).

(B) Enlarged sections of kymograph (boxes) in (A). Scale bar $=10 \mu \mathrm{m}$.

(C) The comparison between TIRF imaging and high resolution iSIM imaging. A mSc::PAR-3;GFP::UTRO zygote at polarization stage is imaged and only GFP.:UTRO channel is shown.

(D) Maximum projected Z stacks of images of mSc::PAR-3;GFP::UTRO zygote, imaged using super-resolution iSIM imaging centered at the cortical region. Anterior to the left. Scale bar $=$ $10 \mu \mathrm{m}$. Yellow box: region enlarged in right column. 


\section{PAR-3 clusters that polarize are subject to higher viscous forces than those that do not}

Our results thus far imply that viscous forces drive the segregation of PAR-3 clusters to the anterior of the zygote to establish polarity. To better understand how this occurs, we examined the diffusion coefficient of engineered PAR-3 oligomers. We found a sharp drop in the diffusion coefficient when oligomer size increased from a 2 mer to a 3 mer (Figures $4 \mathrm{~A}$ ). Slower diffusion of larger oligomers would result in reduced random motion of PAR-3 clusters, facilitating their transport to the anterior by cortical flow.

The diffusion coefficient is directly related to the frictional drag a particle experiences. This relationship is described by the Einstein-Smoluchowski relation

$$
D=\frac{k_{B} T}{f}
$$

Where $D$ is the diffusion constant and $f$ is the frictional drag coefficient, which expresses the viscous drag experienced by a diffusing molecule. Therefore, measurements of the diffusion coefficient provide direct information about the viscous forces a particle is experiencing. We calculated the frictional drag coefficient for each particle in our dataset and found that it was more than 20-fold higher for PAR-3 3mers compared to 2 mers (median $f_{2 \mathrm{mer}}=14.5 \mu \mathrm{g} / \mathrm{s}$, median $f_{3 m e r}=328.5 \mu \mathrm{g} / \mathrm{s}$ for wild-type conditions) (Figure 4B). Thus, PAR-3 3mers (and larger oligomers) are subject to higher viscous forces than 2 mers during polarity establishment. We measured a slightly lower frictional drag for PAR-3 4mers compared to 3mers, but we believe this small unexpected trend is due to measurement error caused by embryo-to-embryo variation (Figure S4).

Another factor that might contribute to the threshold in PAR-3 segregation is the cooperativity between membrane binding lifetime and PAR-3 cluster size. In a polarizing C.elegans embryo, the anterior directed flow is limited to the cortex and does not occur in bulk cytoplasm (Hird and White, 1993; Munro et al., 2004). Therefore, in order to be segregated by cortical flow, proteins must be stably localized to the cortex. PAR-3 achieves this localization by membrane binding through its PDZ-2 domain and/or C-terminal basic domain (Horikoshi et al., 2011; Krahn et al., 2010; Li et al., 2010a; Wu et al., 2007). We observed an average of $\sim 6.8$ seconds membrane lifetime for 2mers, but 25 seconds membrane lifetime for 3mers, 4mers and 6mers (Figure 4C). We did not observe PAR-3 monomers stabilizing on the membrane for more than 1 image frame, consistent with our previous report that a PAR-3 monomeric mutant did not localize to the membrane (Dickinson et al., 2017). The membrane binding lifetime of engineered PAR-3 oligomers increased in concert with the effectiveness of PAR-3 segregation, which suggests that the membrane binding lifetime works in concert with cluster size-dependent changes in diffusion to polarize PAR-3 clusters. 

available under aCC-BY-NC-ND 4.0 International license.
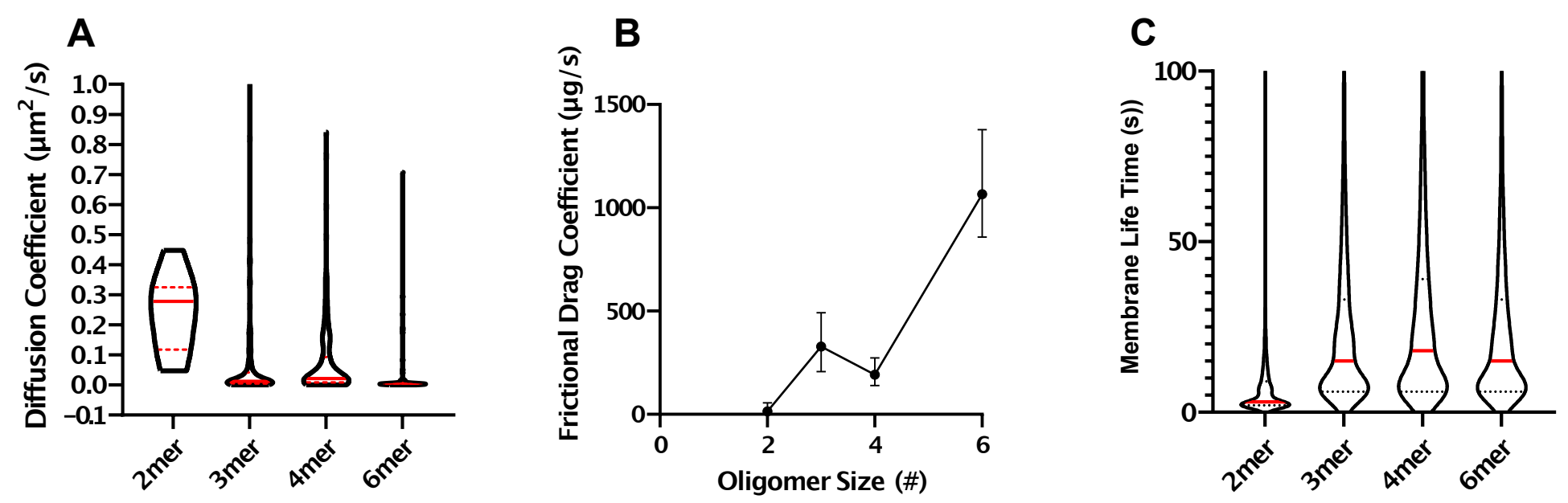
bioRxiv preprint doi: https://doi.org/10.1101/2021.06.07.447386; this version posted June 7, 2021. The copyright holder for this preprint (which was not certified by peer review) is the author/funder, who has granted bioRxiv a license to display the preprint in perpetuity. It is made available under aCC-BY-NC-ND 4.0 International license.

Figure 4. Viscous forces increase sharply for larger PAR-3 oligomers

(A) The diffusion coefficient in engineered PAR-3 strains (wild-type background). Solid lines indicate medians. Dashed lines indicate upper and lower quartiles. Data points pooled from 3 embryos.

(B) Frictional drag coefficients for PAR-3 clusters of different sizes, calculated from the diffusion coefficients of individual PAR-3 particles.

(C) The membrane lifetime of different EOD strains. 


\section{Tracking PAR-3 movement with a novel dual-labeling technique confirms the viscous friction model in an endogenous setting}

Up to this point, we have characterized the diffusive behavior of engineered EOD::PAR-3, which revealed a clear size threshold behavior but represents a somewhat artificial situation. To test whether the size-dependent diffusion behavior of PAR-3 clusters is also true in endogenous conditions, we sought to measure the anomalous parameter and diffusion coefficient of a heterogeneous wild-type PAR-3 population. It is challenging to perform this analysis using fluorescent protein tagged PAR-3, for two reasons: first, there is an inherent bias towards bright signals during particle tracking, and second, the high density of PAR-3 clusters causes challenges in particle tracking, limiting the ability to accurately assemble long tracks for all but the brightest particles. In our previous work (Dickinson et al., 2017), these challenges prevented us from measuring diffusion parameters of endogenously tagged mNeonGreen::PAR-3.

To solve these issues, we developed a new method that allowed us to monitor the diffusion of PAR-3 clusters in an unbiased way while also estimating their sizes. We labeled endogenous HaloTag::PAR-3 with a mixture of two different HaloTag ligand dyes (Grimm et al., 2015, 2016, 2017) at a defined ratio. Each HaloTag molecule binds to a single dye molecule, so that when a mixture of dyes is used for labeling, a mixed population of labeled Halo::PAR-3 molecules results (Figure 5A). By adjusting the ratio of the two dyes, we achieved conditions in which the less-abundant dye was present at single-molecule levels on the cortex, yielding discrete labels that are ideal for tracking. At the same time, the more-abundant dye reveals the cluster size information (Figure 5A).

We imaged dual-labeled Halo::PAR-3 embryos at 20 frames/second on a custom-built TIRF microscope with a dual-view emission path design, allowing simultaneous imaging of both wavelengths. We first analyzed the images of the sparse (red) dye, using the same method as for EOD strains, to measure the anomalous parameter and diffusion coefficient of each track. Then, we found the relative size of each particle by fetching the fluorescent intensity of each particle in the abundant (far-red) channel (Figure 5A). To convert this relative size, in the form of fluorescence intensity, into an estimate of molecules per cluster, we adopted a calibration procedure using the fluorescence intensities of single sparse (red) molecules as an internal standard (Figure 5B, S5 and Methods). In brief, we diluted both HaloTag ligand dyes to single-molecule levels and collected images under identical imaging conditions as those used for the diffusion analysis above; this allowed us to measure the brightness of single far-red dye molecules, which we then used to estimate the number of HaloTag::PAR-3 molecules in each cluster in our sparse/abundant experiments (Figure 5B).

Tracking single endogenous PAR-3 clusters in this way confirmed the trends we found in our experiments with engineered EOD::PAR-3. First, PAR-3 clusters containing more than 3-4 monomers had anomalous diffusion parameters close to 2, indicating that they move in a directed fashion towards the anterior as a consequence of cortical flow (Figure 5C). Smaller clusters had lower values of the anomalous diffusion parameter, indicative of more diffusive motion. Above a size threshold of $\sim 5$ PAR-3 monomers, the anomalous parameter remained constant; thus, above this threshold, the advective transport of PAR-3 molecules by cortical flow 
bioRxiv preprint doi: https://doi.org/10.1101/2021.06.07.447386; this version posted June 7, 2021. The copyright holder for this preprint (which was not certified by peer review) is the author/funder, who has granted bioRxiv a license to display the preprint in perpetuity. It is made available under aCC-BY-NC-ND 4.0 International license.

is efficient regardless of cluster size (Figure 5C). Second, we confirmed the sharp decrease in diffusion coefficient for clusters containing more than a few PAR-3 molecules, indicating that wild-type PAR-3 experiences the same rapid increase in viscous force above a threshold size as our engineered oligomers (Figure 5D). Together, we conclude that a size threshold governs the diffusive behavior and advective transport of wild-type PAR-3 to establish the anterior-posterior axis of the $C$. elegans embryo. 
bioRxiv preprint doi: https://doi.org/10.1101/2021.06.07.447386; this versionposted June 7, 2021. The copyright holder for this preprint (which was not certified by peer review) is the author/funder, who has granted bioRxiva license to display the preprint in perpetuity. It is made available under aCC-BY-NC-ND 4.0 International license.

A
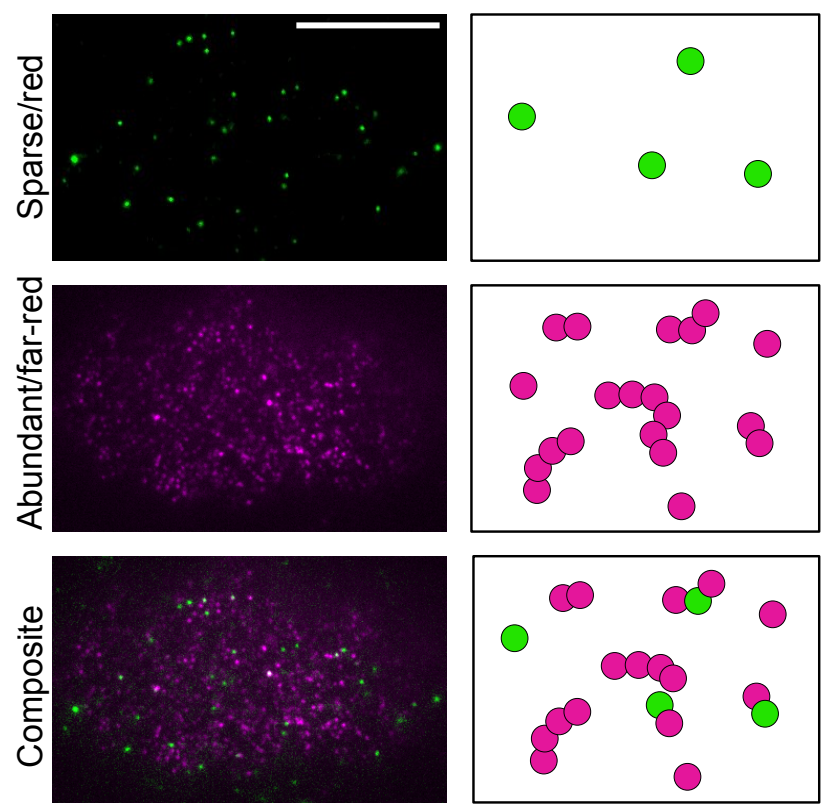

B
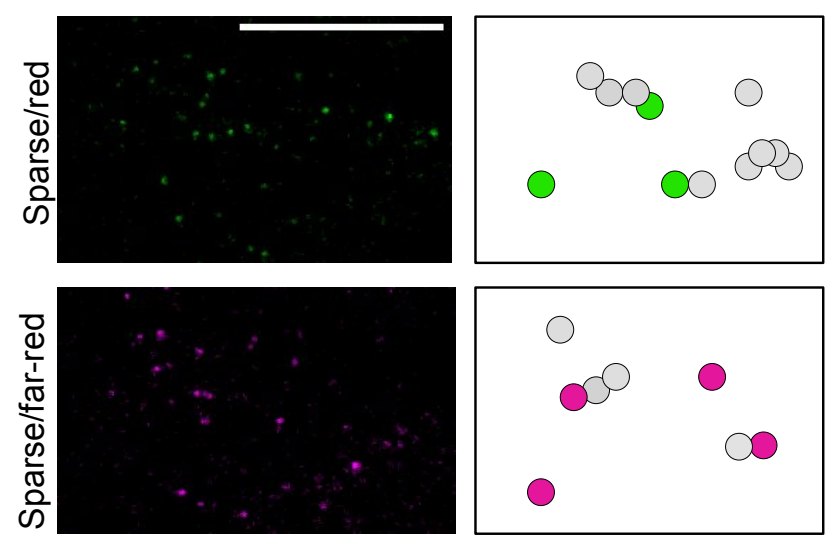

C

Particle

tracking

size info

Size \& tracks correlation

Brightness

Calibration

Calculate

Avg. Intensity

per molecule
Estimated Cluster Size

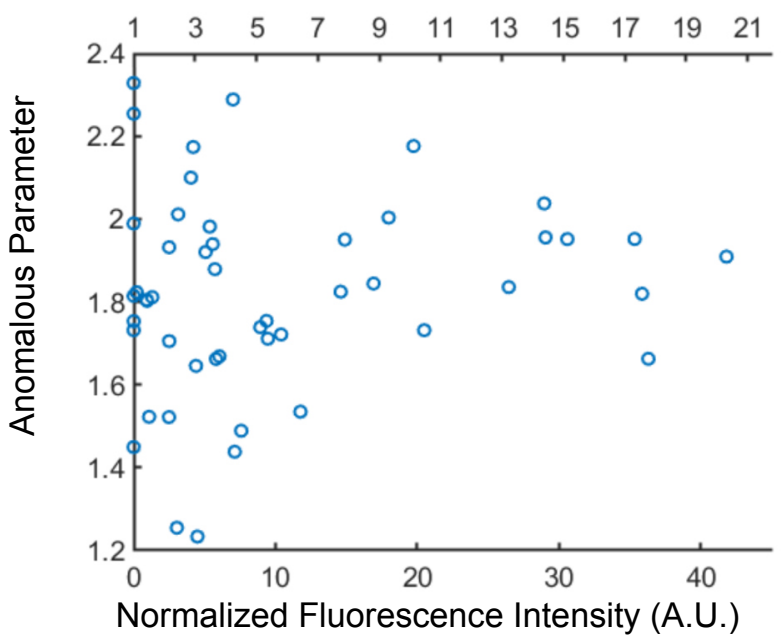

D

Estimated Cluster Size

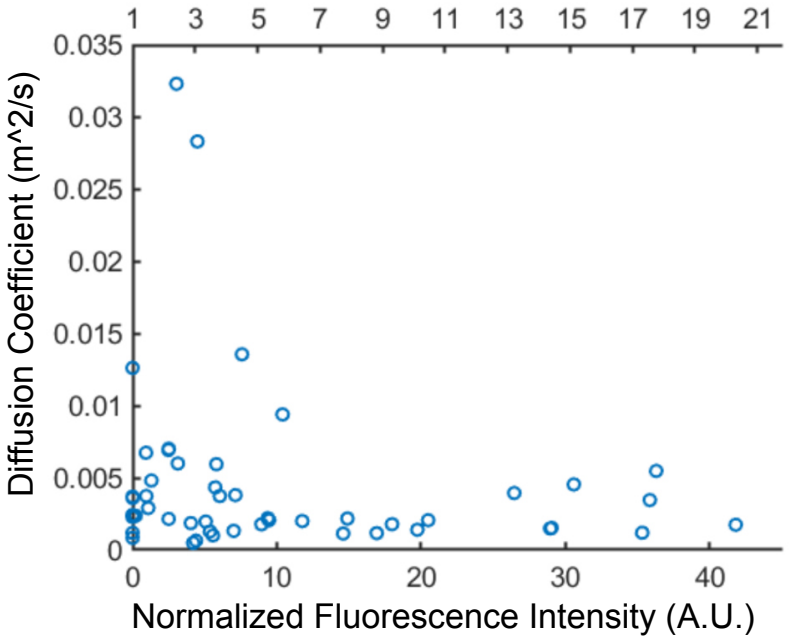


bioRxiv preprint doi: https://doi.org/10.1101/2021.06.07.447386; this version posted June 7, 2021. The copyright holder for this preprint (which was not certified by peer review) is the author/funder, who has granted bioRxiv a license to display the preprint in perpetuity. It is made available under aCC-BY-NC-ND 4.0 International license.

Figure 5. The viscous friction model in an endogenous setting

(A) The TIRF imaging snapshot and cartoon illustration of the dual-labeling experiment. Magenta represents far-red/abundant channel and green represents red/sparse channel (B) The TIRF imaging snapshot and cartoon illustration of the calibration double dilution experiment, where the dyes for both channels are diluted to single molecular level.

(C) The anomalous parameter as a function of size.

(D) The diffusion coefficient as a function of size. 


\section{Discussion}

Cortical flow is a general cell behavior that has been reported in a variety of cell types and serves several different functions (Ananthakrishnan and Ehrlicher, 2007). Here we have studied the biophysical basis for oligomerization-dependent protein transportation by cortical flow. We have used 2 novel methods - engineering PAR-3 size via EODs and dual-labeling of endogenous PAR-3 - to dissect the diffusive behavior of PAR-3 clusters of different sizes. Both approaches revealed a sharp decrease in diffusion coefficient and an increase in anomalous parameters as cluster size increases from two to four monomers, but showed only slight changes in diffusive behavior as clusters grow larger. These experiments indicate that the motion of smaller PAR-3 clusters is dominated by Brownian diffusion, while larger PAR-3 clusters are effectively transported by advective flow. These trends are consistent with our previous observations (Dickinson et al., 2017) but add an additional degree of quantitative precision and raise new questions about the physical and molecular basis for the sharp shift in diffusive parameters at the size threshold.

Our novel dual-labeling strategy can be widely applied for monitoring the dynamics of membrane proteins, as many as $35 \%$ of which may be oligomeric (Goodsell and Olson, 2000). Our approach was inspired by pioneering studies that achieved single-molecule labeling in vivo via low level transgenic expression of the target protein coupled with partial photo-bleaching to tune the levels of fluorescence (Robin et al., 2014), and by single-Molecule Speckle (SiMS) Microscopy via microinjection (Yamashiro and Watanabe, 2017). Dual HaloTag labeling is easier to carry out than these earlier approaches, and additionally allows tracking of sparse labels without sacrificing the ability to visualize the bulk cellular protein. This approach could be applied to other examples of protein oligomerization. For example, E-cadherin forms clusters on the cell membrane that have recently been shown to be crucially important for transmission of forces during tissue morphogenesis (Huebner et al., 2021). Although the dynamics of these clusters have been studied by bulk techniques such as FRAP, a dual-labeling technique could reveal turnover rate and diffusivity, potentially providing new insights into how E-cadherin clustering controls its behavior. As another example, receptor tyrosine kinases (RTKs) are known to be modulated by homo-oligomerization (Schlessinger, 2000), but how oligomerization controls RTK activity is still not understood in detail. A dual-labeling technique, coupled with activity reporters, could provide insight into this question. Finally, we anticipate applying dual-labeling to other oligomeric proteins within the PAR polarity system, including PAR-2 (Arata et al., 2016) and CHIN-1 (Sailer et al., 2015), to determine how clustering affects their dynamics on the plasma membrane.

PAR-3 is a peripheral membrane protein that associates with the lipid bilayer by binding to phosphoinositides (Horikoshi et al., 2011; Krahn et al., 2010; Wu et al., 2007). In vitro, the diffusion of phosphoinositide-binding proteins has been found to follow a remarkably simple relationship in which the viscous drag increases linearly with the number of phospholipid molecules bound by each protein molecule (Knight et al., 2010; Ziemba and Falke, 2013). This relation holds in vitro because 1) the viscosity of the aqueous medium outside the lipid bilayer - in which the protein itself is diffusing - is negligible compared to the viscosity of the lipid 
bilayer itself, and because 2) individual lipids bound by a protein complex are far enough apart that they do not interact with one another within the lipid bilayer. The linear model (Knight et al., 2010; Ziemba and Falke, 2013) predicts that as oligomer size increases from 2 to 3 or from 2 to 6 , viscous drag should increase 1.5 -fold $(3 \div 2)$ and 3 -fold $(6 \div 2)$, respectively. However, in vivo, we observed a much steeper increase in drag: the frictional drag coefficient increased 22 -fold and 73-fold as oligomer size increased from 2 to 3 and from 2 to 6 , respectively. It remains unclear what physical properties of larger PAR-3 oligomers lead to such large increases in viscous friction, but we suggest three possibilities that are not mutually exclusive. First, the viscosity of the cortical cytoplasm might be significantly higher than the buffer used in supported lipid bilayer experiments, which could add a contribution to viscous friction from the cytoplasm in addition to the membrane. Second, the lipids bound by individual PAR-3 monomers within a cluster might interact with one another or nucleate the assembly of a membrane microdomain, which would slow diffusion significantly. Third, larger PAR-3 oligomers might make additional interactions that could affect their conformation or interaction with the membrane.

In regard to this third possibility, previous studies have demonstrated that PAR-3 oligomerization and its binding to PAR-6/aPKC are cooperative (Dickinson et al., 2017). In single cell, single molecule pull-down (sc-SiMPull) experiments, PAR-3 clusters containing 3 or more subunits bound strongly to aPKC/PAR-6, while dimers bound more weakly and monomers associated only at background levels (Dickinson et al., 2017). This observation was in agreement with earlier work showing that punctate PAR-3 and PAR-6/aPKC signals colocalize on the cortex (Beers and Kemphues, 2006; Dickinson et al., 2017; Hung and Kemphues, 1999; Lang and Munro, 2017; Li et al., 2010b). The threshold for PAR-3 binding to PAR-6/aPKC is strikingly consistent with our observed size threshold for PAR-3 clusters to be efficiently segregated by cortical flow, suggesting a possible connection between PAR-3 transportation and aPKC/PAR-6 binding. One hypothesis is that aPKC/PAR- 6 binding could significantly increase the volume of the aPAR complex, making it more susceptible to the viscous friction and thus overcoming random Brownian diffusion. Alternatively, association with PAR-6/aPKC might facilitate membrane binding of PAR-3, either by inducing a PAR-3 conformational change (Chen et al., 2013) or through association with CDC-42 (Joberty et al., 2000) or other partners. Future work will attempt to test whether binding to PAR-6/aPKC is required for the shift in diffusive behavior that we report here.

In our previous study, the cell cycle kinase PLK-1 was shown to negatively regulate PAR-3 oligomerization by phosphorylating residues in the PAR-3 oligomerization domain (Dickinson et al., 2017). In that study, we attempted to isolate constitutive oligomerization mutants of PAR-3 by mutating the two identified PLK-1 target residues to alanine, but were unable to generate stable lines due to highly penetrant lethality and sterility. Consistent with this result, we were unable to isolate EOD::PAR-3 direct fusion lines through CRISPR-mediated insertion of EODs into the PAR-3 locus, which necessitated using the nAb-linking strategy instead. In light of these results, it was surprising that our EOD::nAb; YFP::PAR-3* strains not only are viable, but are healthier than the PAR $-3^{*}$ monomeric mutants. We do not have a clear explanation for this observation. One possibility is that PAR-3 monomers - which are expected to be absent from both the direct EOD::PAR-3* fusion strains and the PLK-1 phosphorylation site mutants, but present in the EOD::nAb; YFP::PAR-3* strains due to the difference in expression level between 
the EOD constructs and PAR-3 - might have a critical, unknown role in development. Alternatively or in addition, phosphorylation of PAR-3 by PLK-1 might regulate crucial PAR-3 interactions other than homoligomerization. One possibility could be that PLK-1 regulates the interaction between PAR-3 and aPKC/PAR-6, given the central role of aPKC in establishing polarized cell behavior. These hypotheses represent interesting areas for further investigation.

\section{Acknowledgments}

We thank Luke Lavis for sharing JaneliaFluor dyes prior to publication, and Edwin Munro, Jeanne Stachowiak, John Wallingford and members of the Dickinson lab for helpful discussions and comments on the manuscript. This work was supported by a Provost's Graduate Education Fellowship from the University of Texas at Austin (YC), by a research grant from the Mallinckrodt foundation (DJD), and by NIH R00 GM115964 and R01 GM138443 (DJD). DJD is a CPRIT Scholar supported by the Cancer Prevention and Research Institute of Texas. Some strains were provided by the Caenorhabditis Genetics Center, which is funded by the NIH Office of Research Infrastructure Programs [P40 OD010440].

\section{Conflict of Interest}

The authors declare that they have no conflicts of interest with the contents of this article.

\section{Author Contributions}

YC and DJD designed the experiments. DJD designed and built the TIRF microscope used for dual-labeling experiments. YC performed the experiments. YC analyzed the data with input from DJD. DJD supervised the project and secured funding. YC and DJD co-wrote the manuscript.

\section{Methods}

\section{KEY RESOURCE TABLE}

The Key Resource Table is provided as a supplemental file.

\section{CONTACT FOR REAGENT AND RESOURCE SHARING}

Requests for resources and further information should be directed and will be fulfilled by the Lead Contact, Daniel J. Dickinson (daniel.dickinson@austin.utexas.edu).

\section{EXPERIMENTAL MODEL AND SUBJECT DETAILS}


All C. elegans strains were fed with OP50 and maintained on standard NGM growth medium. All strains were kept in a $20^{\circ} \mathrm{C}$ incubator unless noted otherwise. Embryos were examined before sex can be determined, however, most of embryos were likely to be hermaphrodites because the spontaneous occurance of male without mating is rare.

All genetic modifications to the $\mathrm{C}$. elegans genome were made using protocols previously published by our laboratory (Dickinson et al., 2013, 2015). In brief, we used NEBuilder HiFi DNA Assembly to generate a plasmid construct that contains the homologous repair template with genome modifications and a selectable marker, flanked by 500-1500 bp of unmodified genomic homology arms. A repair template construct, a Cas9-sgRNA expressing vector, and a vector expressing extrachromosomal array fluorescent markers were co-injected into the syncytial gonads of young adult hermaphrodites. DNA repair by homologous recombination was triggered by Cas 9 cleavage of the $C$. elegans genome, which allows the incorporation of modified repair template via homologous recombination. Knock-in animals were selected from the F2 progeny of injected animals using hygromycin selection and a phenotypic marker. After each knock-in strain was isolated, the selectable marker was excised by Cre-Lox recombination.

\section{METHOD DETAILS}

\section{TIRF Microscopy}

Adult C.elegans carrying eggs were dissected in a drop of egg buffer on polylysine-coated coverslips. The embryos were gently flattened by mounting with $22.8 \mathrm{~mm}$ beads (Whitehouse scientific, Chester, UK) as spacers. Dual-labeling experiments (Figure 5) were carried out on a custom-built TIRF microscope (see below). All other TIRF images were acquired using a Nikon Eclipse Ti-2 microscope equipped with a 100X, 1.49 NA objective; a Photometrics Prime 95B camera; and an iLas2 circular TIRF illuminator (Roper scientific, E' vry, France). TIRF images were magnified by a $1.5 \mathrm{X}$ tube lens before being collected by camera chip. The TIRF illuminator was operated in ellipse mode for acquiring images of whole embryos. mNG was excited using a $488 \mathrm{~nm}$ laser; YFP was excited using a $505 \mathrm{~nm}$ laser; mScarlet and JF585 were excited using a $561 \mathrm{~nm}$ laser; and JF646 was excited using a $637 \mathrm{~nm}$ laser.

\section{Super-resolution iSIM imaging}

Adult C.elegans carrying eggs were dissected in a drop of egg buffer on polylysine-coated coverslips. The embryos were gently flattened by mounting with $22.8 \mathrm{~mm}$ beads (Whitehouse scientific, Chester, UK) as spacers. Images were acquired using a Nikon Eclipse Ti-2 microscope equipped with a 100X, 1.49 NA objective; a Photometrics Prime BSI camera; an OptoSpin filter wheel (CAIRN Research, Kent, England), and an vt-iSIM super-resolution confocal scan head (VisiTech international, Sunderland, UK). Confocal images were magnified by a $1.5 \mathrm{X}$ tube lens before being collected by camera chip. During image acquisition, the focal plane was centered at the cortex, and along with 2 slices above and below, 0.25 um per slice step, 5 total slices were collected. GFP was excited using a $488 \mathrm{~nm}$ laser and $\mathrm{mScarlet}$ was excited using a $561 \mathrm{~nm}$ laser. 
Imaging processing and display

The high-resolution iSIM images (Figure 1C-D and S3D) were processed through FIJI to generate $Z$ maximum projection images. The PAR-3 channel (Figure 1D) was processed using the RF denoise command in FIJI (theta $=10$ ) after maximum projection. Following these operations, brightness and contrast were adjusted for visibility of the signals. No other image manipulations were performed.

To generate kymographs (Figures 1A), we extracted a 15 pixel wide strip at the center of the imaged embryo image and stacked these strips on top of one another using the "montage" command in FIJI.

\section{Particle Image Velocimetry quantification}

The quantify the coupled motion of PAR-3 and actin filaments network (Figure S1B), we applied velocimetry (PIV) to the mSc::PAR-3 and GFP::UTRO image channel using the PIVlab MATLAB plugin (Thielicke and Stamhuis, 2014). For mSc::PAR-3 channel: Images were pre-processed with the high-pass filter $=50$, Wiener2 denoise filter $=5$. The contrast was manually adjusted. Other preprocessing filters were disabled. Flow detection used the default FFT phase-space algorithm with 3 passes (window sizes 64, 32 and 16 pixels) and linear window deformation. Post-processing was done with velocity limits drawn manually to exclude outliers. Vectors that were rejected by these filters were replaced by interpolation. For Utrophin channel: Images were pre-processed with the high-pass filter $=400$, Wiener2 denoise filter $=2$. Other processing methods were the same as for mSc::PAR-3 channel.

\section{Pearson's correlation}

To quantify the correlation of velocity in PAR-3 and cortex (Figure S1C), the data was organized to a three dimensional matrix, with size of ( $x$-dimension, $y$-dimension, 2 (x-vector/y-vector)). Pearson's correlation was calculated by MATLAB Command: corrcoef (UTRO velocity matrix, PAR-3 velocity matrix).

To quantify the colocalization of PAR-3 and actin, we imported the image sequence of mSc::PAR-3 and GFP::UTRO into the matlab as a numerical matrix. Data was organized to a three dimensional matrix, with size of ( $x$-pixel position, y-pixel position, t-time resolution). Pearson's correlation was calculated by MATLAB Command: corrcoef (UTRO velocity matrix, PAR-3 velocity matrix).

\section{Cell polarization quantification and fluorescence measurements}

To quantify the polarization ability of our EOD strains, ovals matching the shape of the anterior half and posterior half of the embryo were drawn and the mean intensity per pixel was quantified using the Measure command in FIJI. For each embryo, the anterior intensity is normalized to 1 and the ratio of posterior intensity/anterior intensity was calculated.

\section{Embryo lethality and sterility quantification}

A single young adult was picked to a new plate. After $12 \mathrm{hrs}$, which allows the adult to lay eggs, the adult was removed from the plate. After another $24 \mathrm{hrs}$ during which the eggs were allowed 
to hatch, any unhatched embryos and hatched larvae were counted and embryonic lethality was calculated. The plate was maintained for additional 3 days for counting the number of fertile adults and sterile adults, which is used for calculating adult sterility.

\section{Sc-SiMPull and photobleaching step counting}

We exactly followed the protocol in Dickinson et al., 2017. For reagents, experimental procedures, and data analysis software, please see Dickinson et al., 2017.

\section{PAR-3 particle tracking and motion analysis}

We used Utrack (Jaqaman et al., 2008) to track the motion of PAR-3 particles in TIRF images. For particle detection, we used the point source detection algorithm with alpha $=0.01$ and other parameters as default. For the particle tracking step, we used Brownian + directed motion mode with minimum track length $=2$. From our experience, we found the quality of tracking is greatly enhanced by closing the gaps during the linking step. However, since the PAR-3 clusters are so dense on the cortical plane (except in dual-labeling experiments), we can only allow a maximum of 3 frames gap-to-close without introducing false linking. Therefore, we used gap-to-close $=3$ for tracking PAR-3 in EOD strains and gap-to-close = 10 for tracking PAR-3 in dual-labeling experiments.

\section{MSD plot generation and calculations for diffusion coefficient and anomalous parameter}

The PAR-3 tracks information generated by Utrack was reformatted for the 'msdanalyzer' MATLAB function, and the results were processed by msdanalyzer' for generating MSD curves (Tarantino et al., 2014). The curve is fitted to $y=a x^{\wedge} n$ using the open-source 'ezyfit' plugin in MATLAB, where Anomalous parameter $=n$ and Diffusion coefficient $=a / 4$.

Before the anomalous parameter and diffusion coefficient were plotted, MSD plots with curve fitting $R<0.98$ were removed. This choice of $R$ value cutoff affects the noise level of the data but not the overall trends we report (Figure S2A-B). We also eliminated tracks that are less than 10 frames, or 0.5 seconds, to prevent a falsely high $\mathrm{R}$ value due to insufficient data points. The choice of minimum track length affects noise levels of the MSD plots, but does not change the overall distribution of MSD curves and the averaged MSD equation.

\section{ezyfit code modified for $\mathbf{R}$ value}

During the curve fitting using the original version of ezyfit, some fits reported an $\mathrm{R}$ value $<0$, which is mathematically impossible. Therefore, we corrected the error in the code for calculating the $R$ value (Kvalseth, 1985).

Original code:

$$
\begin{aligned}
& \text { SSreg }=\operatorname{sum}\left(\left(y \_f i t-\operatorname{mean}(y)\right) \cdot{ }^{\wedge} 2\right) \% \text { regression sum of squares } \\
& \text { SStot }=\operatorname{sum}\left((y-\operatorname{mean}(y)) \cdot{ }^{\wedge} 2\right) \% \text { total sum of squares }
\end{aligned}
$$

Modified version:

$$
\begin{aligned}
& \text { ssreg = sum(abs(y_fit-y).^2); } \\
& \text { sstot = sum(abs(y-mean(y)).^2); } \\
& \text { f.r = } 1 \text { - ssreg/sstot; }
\end{aligned}
$$




\section{Membrane lifetime quantification}

To quantify the membrane lifetime of EOD::PAR-3 clusters (Figure 4C), we tracked YFP::PAR-3 clusters using Utrack. The Utrack output was translated into a more readable format using a custom-written MATLAB script, and the track length, in frames, was extracted. The membrane life time was calculated from the track length as following:

Membrane lifetime $=$ Imaging time interval (seconds) ${ }^{*}$ Track Length (frames)

\section{RNA interference}

RNA interference targeting m/c-4 to eliminate cortical flow was performed by injection.We amplified $1 \mathrm{~kb}$ of $\mathrm{m} / \mathrm{c}-4$ from cDNA using primers that added T7 promoters to both ends of the amplified fragment. dsRNA was then synthesized using the T7 Ribomax kit (Promega) as instructed by the manufacturer. $1 \mathrm{mg} / \mathrm{mL} \mathrm{m} / \mathrm{c}-4$ dsRNA was injected into young adults in either GFP::Utrophin background or EOD;PAR-3 background, and the worms were dissected 24-28 hrs later for embryo imaging.

\section{HaloTag ligand dye feeding and Dual-labeling experiments}

JaneliaFluor Dyes (gifts from Luke Lavis) were dissolved in acetonitrile to $1 \mathrm{mM}$, dispensed into $2 \mu \mathrm{L}$ aliquots in PCR tubes, dried under vacuum and stored at $-20 \mathrm{C}$ in a desiccator upon receipt. Before use, $2 \mu \mathrm{L}$ of DMSO was used to dissolve each single-use aliquot. $1 \mathrm{~mL}$ of OP50 liquid culture was spun down and resuspended in $100 \mu \mathrm{L} \mathrm{S}$ medium $(150 \mathrm{mM} \mathrm{NaCl}, 1 \mathrm{~g} / \mathrm{L}$ $\mathrm{K} 2 \mathrm{HPO} 4,6 \mathrm{~g} / \mathrm{L} \mathrm{KH} 2 \mathrm{PO} 4,5 \mu \mathrm{g} / \mathrm{L}$ cholesterol, $10 \mathrm{mM}$ potassium citrate $\mathrm{pH}$ 6.0, $3 \mathrm{mM} \mathrm{CaCl} 2,3$ $\mathrm{mM} \mathrm{MgCl} 2,65 \mu \mathrm{M}$ EDTA, $25 \mu \mathrm{M}$ FeSO4, $10 \mu \mathrm{M} \mathrm{MnCl} 2,10 \mu \mathrm{M}$ ZnSO4, $1 \mu \mathrm{M}$ CuSO4) for each dye. Dissolved dye was added to the $S$ medium-bacteria mixture. For the dual-labeling experiment, $2 \mu \mathrm{L}$ of JF585-medium-bateria mix was added to $100 \mu \mathrm{L}$ JF646-medium-bateria mix. For the double dilution experiment, a separate $100 \mu \mathrm{L}$ medium-bacteria mix was made, and $2 \mu \mathrm{L}$ of JF585-medium-bateria mix and $1 \mu \mathrm{L}$ of JF646-medium-bateria mix was added to the 100 $\mu \mathrm{L}$ medium-bacteria mix. $30 \mu \mathrm{L}$ of dye(s)-medium-bateria mix was dispensed into wells of a 96-well plate with round-bottom wells, and 20-30 L4-stage worms were picked into each well. Worms were grown at $20^{\circ} \mathrm{C}$, with shaking at $230 \mathrm{rpm}$, for $16-24 \mathrm{hrs}$ before imaging. The final JaneliaFluor dye concentration used for feeding was $15 \mathrm{uM}$.

\section{Micromirror TIRF imaging}

Adult C.elegans carrying eggs were dissected in a drop of egg buffer on polylysine-coated coverslips. The embryos were gently flattened by mounting with $22.8 \mathrm{~mm}$ beads (Whitehouse scientific, Chester, UK) as spacers. TIRF images were acquired using a custom-built TIRF microscope equipped with an Olympus APON 60X, 1.49 NA objective; a Photometrics Prime 95B camera; and a dual-view emission path design. This microscope uses a micromirror illumination path design that is optimal for imaging multiple wavelengths simultaneously (Friedman and Gelles, 2015; Friedman et al., 2006). After collection of fluorescence emission by the objective lens, an image was formed using a tube lens (Edmund Optics) with a $400 \mathrm{~mm}$ focal length, resulting in $133.33 \mathrm{X}$ magnification. The red and far-red channels were split using a T635lpxr dichroic mirror (Chroma), passed through a set of relay lenses, and collected side-by-side on the camera chip. The relay lenses introduced an additional 1.2X magnification, 
so that the total system magnification was 160X. JF585 and JF646 were simultaneously excited using $5 \mathrm{~mW} 561 \mathrm{~nm}$ laser and 50mW $637 \mathrm{~nm}$ laser.

\section{Dual-labeling experiments data analysis}

We calibrated JF646 intensity using control experiments in which both JF585 and JF646 were diluted to single-molecule levels. As expected, the measured fluorescence intensities of single dye molecules followed a log-normal distribution (Figure S5A-B) (Mutch et al., 2007). The measured single-fluorophore intensities varied from embryo to embryo, which is not surprising and likely reflects variations in mounting and eggshell thickness; importantly, however, the intensities of the red and far-red channels varied in tandem (Figure S5A). We therefore adopted the following calibration procedure, in which we used the red dye intensity as an internal standard, since it is present at single-molecule levels in both sets of experiments. First, we normalized our double-dilution datasets to the red dye intensity. Normalization was done in log space, to account for the log-normal shape of the intensity distribution, and was effective at correcting for embryo-to-embryo variations in fluorescence intensity (Figure S5A-C). Second, we calculated the ratio of the average far-red dye intensity to the average red dye intensity (Figure S5D). Third, the intensity of red dyes in each sparse/abundant dual-labeling experiment was measured, and the intensities were normalized to the mean red dye intensity to allow comparison between embryos (Figure S5E-H). Fourth, from the calibrated red/far-red intensity ratio, an expected mean far-red intensity per molecule was calculated. Finally, this calculated far-red intensity per molecule was used to estimate the number of far-red labeled Halo::PAR-3 molecules in each tracked cluster (Figure 5C-D, top x-axes). This calibration procedure accounts for the differences in global fluorescence intensity between embryos, presumably caused by the differences in laser penetration depth and eggshell thickness, and allows us to estimate the number of PAR-3 molecules per cluster.

The cluster size information of PAR-3 labeled by red fluorophore JF585 was acquired by a custom-written MATLAB script. This script draws a box in the far-red channel at the location where the red signal is located, and calculates the fluorescence intensity in the far-red channel by subtracting local background from a larger box. To account for the brightness variation across different embryos, we followed the procedure that is summarized in the preceding paragraph. We first calculated the maximum likelihood estimate of single JF585 fluorophore intensity in log normal distribution by using matlab function 'mle(data, 'dist', 'LogNormal')'; Second, single molecular red and farred intensities were transformed into log scale; Third, log scale JF585 intensities were calibrated to 1 by subtracting an offset value, and the log scale JF646 intensity was calibrated according to the calibration of red channel by subtracting the same offset value; Fourth, all double dilution datasets were pooled together and the mean intensity of single JF646 fluorophore was calculated; Finally, a ratio of single JF585 intensity and single JF646 intensity was calculated and was used to estimate cluster size in the dual-labeling experiment. 
bioRxiv preprint doi: https://doi.org/10.1101/2021.06.07.447386; this version posted June 7, 2021. The copyright holder for this preprint (which was not certified by peer review) is the author/funder, who has granted bioRxiv a license to display the preprint in perpetuity. It is made available under aCC-BY-NC-ND 4.0 International license.

Yiran Chang \& Daniel J. Dickinson 2021, p. 29 


\section{References}

Allen, N.S., and Allen, R.D. (1978). Cytoplasmic Streaming in Green Plants. Annu Rev Biophys Bio 7 , 497-526.

Ananthakrishnan, R., and Ehrlicher, A. (2007). The Forces Behind Cell Movement. Int J Biol Sci 3, 303-317.

Arata, Y., Hiroshima, M., Pack, C.-G., Ramanujam, R., Motegi, F., Nakazato, K., Shindo, Y., Wiseman, P.W., Sawa, H., Kobayashi, T.J., et al. (2016). Cortical Polarity of the RING Protein PAR-2 Is Maintained by Exchange Rate Kinetics at the Cortical-Cytoplasmic Boundary. Cell Reports 16, 2156-2168.

Beers, M., and Kemphues, K. (2006). Depletion of the co-chaperone CDC-37 reveals two modes of PAR-6 cortical association in C. elegans embryos. Development 133, 3745-3754.

Benton, R., and Johnston, D.St. (2003). A Conserved Oligomerization Domain in Drosophila

Bazooka/PAR-3 Is Important for Apical Localization and Epithelial Polarity. Curr Biol 13, 1330-1334.

Bolten, M., Delley, C.L., Leibundgut, M., Boehringer, D., Ban, N., and Weber-Ban, E. (2016). Structural

Analysis of the Bacterial Proteasome Activator Bpa in Complex with the 20S Proteasome. Structure 24, 2138-2151.

Boudko, S.P., Sasaki, T., Engel, J., Lerch, T.F., Nix, J., Chapman, M.S., and Bächinger, H.P. (2009). Crystal Structure of Human Collagen XVIII Trimerization Domain: A Novel Collagen Trimerization Fold. J Mol Biol 392, 787-802.

Büttner, C.R., Chechik, M., Ortiz-Lombardía, M., Smits, C., Ebong, I.-O., Chechik, V., Jeschke, G., Dykeman, E., Benini, S., Robinson, C.V., et al. (2012). Structural basis for DNA recognition and loading into a viral packaging motor. Proc National Acad Sci 109, 811-816.

Chen, S., Chen, J., Shi, H., Wei, M., Castaneda-Castellanos, D.R., Bultje, R.S., Pei, X., Kriegstein, A.R., Zhang, M., and Shi, S.-H. (2013). Regulation of Microtubule Stability and Organization by Mammalian Par3 in Specifying Neuronal Polarity. Dev Cell 24, 26-40.

Chik, J.K., Moiseeva, V., Goel, P.K., Meinen, B.A., Koldewey, P., An, S., Mellone, B.G., Subramanian, L., and Cho, U.-S. (2019). Structures of CENP-C cupin domains at regional centromeres reveal unique patterns of dimerization and recruitment functions for the inner pocket. J Biol Chem 294, 14119-14134. Cuenca, A.A., Schetter, A., Aceto, D., Kemphues, K., and Seydoux, G. (2003). Polarization of the C. elegans zygote proceeds via distinct establishment and maintenance phases. Development 130, 1255-1265.

Dickinson, D.J., Schwager, F., Pintard, L., Gotta, M., and Goldstein, B. (2017). A Single-Cell Biochemistry Approach Reveals PAR Complex Dynamics during Cell Polarization. Dev Cell 42, 416-434.e11.

Drulyte, I., Obajdin, J., Trinh, C.H., Kalverda, A.P., Kamp, M.W. van der, Hemsworth, G.R., and Berry, A. (2019). Crystal structure of the putative cyclase IdmH from the indanomycin nonribosomal peptide synthase/polyketide synthase. lucrj 6, 1120-1133.

Friedman, L.J., and Gelles, J. (2015). Multi-wavelength single-molecule fluorescence analysis of transcription mechanisms. Methods 86, 27-36.

Friedman, L.J., Chung, J., and Gelles, J. (2006). Viewing Dynamic Assembly of Molecular Complexes by Multi-Wavelength Single-Molecule Fluorescence. Biophys J 91, 1023-1031.

Goehring, N.W., Trong, P.K., Bois, J.S., Chowdhury, D., Nicola, E.M., Hyman, A.A., and Grill, S.W. (2011). Polarization of PAR Proteins by Advective Triggering of a Pattern-Forming System. Science 334, 1137-1141.

Goodsell, D.S., and Olson, A.J. (2000). STRUCTURAL SYMMETRY AND PROTEIN FUNCTION. Annu Rev Bioph Biom 29, 105-153.

Grimm, J.B., English, B.P., Chen, J., Slaughter, J.P., Zhang, Z., Revyakin, A., Patel, R., Macklin, J.J., Normanno, D., Singer, R.H., et al. (2015). A general method to improve fluorophores for live-cell and single-molecule microscopy. Nature Methods 12, 244-250, 3 p following 250.

Grimm, J.B., English, B.P., Choi, H., Muthusamy, A.K., Mehl, B.P., Dong, P., Brown, T.A., Lippincott-Schwartz, J., Liu, Z., Lionnet, T., et al. (2016). Bright photoactivatable fluorophores for single-molecule imaging. Nature Methods 13, 985-988.

Grimm, J.B., Muthusamy, A.K., Liang, Y., Brown, T.A., Lemon, W.C., Patel, R., Lu, R., Macklin, J.J., Keller, P.J., Ji, N., et al. (2017). A general method to fine-tune fluorophores for live-cell and in vivo imaging.

Nature Methods 14, 987-994.

Gross, P., Kumar, K.V., Goehring, N.W., Bois, J.S., Hoege, C., Jülicher, F., and Grill, S.W. (2019). Guiding 
self-organized pattern formation in cell polarity establishment. Nat Phys 15, 293-300.

Hird, S.N., and White, J.G. (1993). Cortical and cytoplasmic flow polarity in early embryonic cells of Caenorhabditis elegans. J Cell Biology 121, 1343-1355.

Hong, Y. (2018). aPKC: the Kinase that Phosphorylates Cell Polarity. F1000research 7, F1000 Faculty Rev-903.

Horikoshi, Y., Hamada, S., Ohno, S., and Suetsugu, S. (2011). Phosphoinositide Binding by Par-3 Involved in Par-3 Localization. Cell Struct Funct 36, 97-102.

Huang, P.-S., Oberdorfer, G., Xu, C., Pei, X.Y., Nannenga, B.L., Rogers, J.M., DiMaio, F., Gonen, T., Luisi, B., and Baker, D. (2014). High thermodynamic stability of parametrically designed helical bundles.

\section{Science 346, 481-485.}

Huebner, R.J., Malmi-Kakkada, A.N., Sarikaya, S., Weng, S., Thirumalai, D., and Wallingford, J.B. (2021). Mechanical heterogeneity along single cell-cell junctions is driven by lateral clustering of cadherins during vertebrate axis elongation. Elife 10, e65390.

Hung, T.J., and Kemphues, K.J. (1999). PAR-6 is a conserved PDZ domain-containing protein that colocalizes with PAR-3 in Caenorhabditis elegans embryos. Dev Camb Engl 126, 127-135.

Jaqaman, K., Loerke, D., Mettlen, M., Kuwata, H., Grinstein, S., Schmid, S.L., and Danuser, G. (2008). Robust single-particle tracking in live-cell time-lapse sequences. Nat Methods 5, 695-702.

Joberty, G., Petersen, C., Gao, L., and Macara, I.G. (2000). The cell-polarity protein Par6 links Par3 and atypical protein kinase $\mathrm{C}$ to Cdc42. Nat Cell Biol 2, 531-539.

Knight, J.D., Lerner, M.G., Marcano-Velázquez, J.G., Pastor, R.W., and Falke, J.J. (2010). Single Molecule Diffusion of Membrane-Bound Proteins: Window into Lipid Contacts and Bilayer Dynamics. Biophys J 99, 2879-2887.

Krahn, M.P., Klopfenstein, D.R., Fischer, N., and Wodarz, A. (2010). Membrane Targeting of Bazooka/PAR-3 Is Mediated by Direct Binding to Phosphoinositide Lipids. Curr Biol 20, 636-642. Kvalseth, T.O. (1985). Cautionary Note about R 2. Am Statistician 39, 279.

Lang, C.F., and Munro, E. (2017). The PAR proteins: from molecular circuits to dynamic self-stabilizing cell polarity. Development 144, 3405-3416.

Lee, N., Patrick, J.W., and Masson, M. (1968). Crystalline L-ribulose 5-phosphate 4-epimerase from Escherichia coli. J Biological Chem 243, 4700-4705.

Lénárt, P., Bacher, C.P., Daigle, N., Hand, A.R., Eils, R., Terasaki, M., and Ellenberg, J. (2005). A contractile nuclear actin network drives chromosome congression in oocytes. Nature 436, 812-818. $\mathrm{Li}, \mathrm{B} ., \mathrm{Kim}, \mathrm{H}$., Beers, M., and Kemphues, K. (2010a). Different domains of C. elegans PAR-3 are required at different times in development. Dev Biol 344, 745-757.

Li, C., Chai, Y., Song, H., Weng, C., Qi, J., Sun, Y., and Gao, G.F. (2019). Crystal Structure of African Swine Fever Virus dUTPase Reveals a Potential Drug Target. Mbio 10, e02483-19.

Li, J., Kim, H., Aceto, D.G., Hung, J., Aono, S., and Kemphues, K.J. (2010b). Binding to PKC-3, but not to PAR-3 or to a conventional PDZ domain ligand, is required for PAR-6 function in C. elegans. Dev Biol 340, 88-98.

Luo, Y., Samuel, J., Mosimann, S.C., Lee, J.E., Tanner, M.E., and Strynadka, N.C.J. (2001). The Structure of I-Ribulose-5-Phosphate 4-Epimerase: An Aldolase-like Platform for Epimerization $\dagger, \ddagger$.

Biochemistry-Us 40, 14763-14771.

Mayer, M., Depken, M., Bois, J.S., Jülicher, F., and Grill, S.W. (2010). Anisotropies in cortical tension reveal the physical basis of polarizing cortical flows. Nature 467, 617-621.

Mehta, A. (2001). Myosin learns to walk. J Cell Sci 114, 1981-1998.

Mittasch, M., Gross, P., Nestler, M., Fritsch, A.W., Iserman, C., Kar, M., Munder, M., Voigt, A., Alberti, S., Grill, S.W., et al. (2018). Non-invasive perturbations of intracellular flow reveal physical principles of cell organization. Nat Cell Biol 20, 344-351.

Mizuno, K., Suzuki, A., Hirose, T., Kitamura, K., Kutsuzawa, K., Futaki, M., Amano, Y., and Ohno, S. (2003). Self-association of PAR-3-mediated by the Conserved N-terminal Domain Contributes to the Development of Epithelial Tight Junctions*. J Biol Chem 278, 31240-31250.

Munro, E., Nance, J., and Priess, J.R. (2004). Cortical Flows Powered by Asymmetrical Contraction Transport PAR Proteins to Establish and Maintain Anterior-Posterior Polarity in the Early C. elegans Embryo. Dev Cell 7, 413-424.

Mutch, S.A., Fujimoto, B.S., Kuyper, C.L., Kuo, J.S., Bajjalieh, S.M., and Chiu, D.T. (2007). Deconvolving Single-Molecule Intensity Distributions for Quantitative Microscopy Measurements. Biophys J 92, 2926-2943. 
Parsons, J.F., Lim, K., Tempczyk, A., Krajewski, W., Eisenstein, E., and Herzberg, O. (2002). From structure to function: Yrbl from Haemophilus influenzae (HI1679) is a phosphatase. Proteins Struct Funct Bioinform 46, 393-404.

Robin, F.B., McFadden, W.M., Yao, B., and Munro, E.M. (2014). Single-molecule analysis of cell surface dynamics in Caenorhabditis elegans embryos. Nat Methods 11, 677-682.

Rodriguez, J., Peglion, F., Martin, J., Hubatsch, L., Reich, J., Hirani, N., Gubieda, A.G., Roffey, J., Fernandes, A.R., Johnston, D.S., et al. (2017). aPKC Cycles between Functionally Distinct PAR Protein Assemblies to Drive Cell Polarity. Dev Cell 42, 400-415.e9.

Sailer, A., Anneken, A., Li, Y., Lee, S., and Munro, E. (2015). Dynamic Opposition of Clustered Proteins Stabilizes Cortical Polarity in the C. elegans Zygote. Dev Cell 35, 131-142.

Santiago-Frangos, A., Fröhlich, K.S., Jeliazkov, J.R., Małecka, E.M., Marino, G., Gray, J.J., Luisi, B.F., Woodson, S.A., and Hardwick, S.W. (2019). Caulobacter crescentus Hfq structure reveals a conserved mechanism of RNA annealing regulation. Proc National Acad Sci 116, 201814428.

Schlessinger, J. (2000). Cell Signaling by Receptor Tyrosine Kinases. Cell 103, 211-225.

Shelton, C.A., Carter, J.C., Ellis, G.C., and Bowerman, B. (1999). The Nonmuscle Myosin Regulatory Light Chain Gene mlc-4 Is Required for Cytokinesis, Anterior-Posterior Polarity, and Body Morphology during Caenorhabditis elegans Embryogenesis. J Cell Biology 146, 439-451.

Sun, L., Young, L.N., Zhang, X., Boudko, S.P., Fokine, A., Zbornik, E., Roznowski, A.P., Molineux, I.J., Rossmann, M.G., and Fane, B.A. (2014). Icosahedral bacteriophage $\Phi \times 174$ forms a tail for DNA transport during infection. Nature 505, 432-435.

Tarantino, N., Tinevez, J.-Y., Crowell, E.F., Boisson, B., Henriques, R., Mhlanga, M., Agou, F., Israël, A., and Laplantine, E. (2014). TNF and IL-1 exhibit distinct ubiquitin requirements for inducing NEMO-IKK supramolecular structuresNEMO-IKK recruitment to supramolecular structures. J Cell Biology 204, 231-245.

Thielicke, W., and Stamhuis, E.J. (2014). PIVlab - Towards User-friendly, Affordable and Accurate Digital Particle Image Velocimetry in MATLAB. J Open Res Softw 2, e30.

Thomson, A.R., Wood, C.W., Burton, A.J., Bartlett, G.J., Sessions, R.B., Brady, R.L., and Woolfson, D.N. (2014). Computational design of water-soluble $\alpha$-helical barrels. Science 346, 485-488.

Tse, Y.C., Werner, M., Longhini, K.M., Labbe, J.-C., Goldstein, B., and Glotzer, M. (2012). RhoA activation during polarization and cytokinesis of the early Caenorhabditis elegans embryo is differentially dependent on NOP-1 and CYK-4. Mol Biol Cell 23, 4020-4031.

Veesler, D., Blangy, S., Lichière, J., Ortiz-Lombardía, M., Tavares, P., Campanacci, V., and Cambillau, C. (2010). Crystal structure of Bacillus subtilis SPP1 phage gp23.1, a putative chaperone. Protein Sci 19, 1812-1816.

Wang, S., Tang, N.H., Lara-Gonzalez, P., Zhao, Z., Cheerambathur, D.K., Prevo, B., Chisholm, A.D., Desai, A., and Oegema, K. (2017a). A toolkit for GFP-mediated tissue-specific protein degradation in C. elegans. Development 144, dev.150094.

Wang, S.-C., Low, T.Y.F., Nishimura, Y., Gole, L., Yu, W., and Motegi, F. (2017b). Cortical forces and CDC-42 control clustering of PAR proteins for Caenorhabditis elegans embryonic polarization. Nat Cell Biol 19, 988-995.

Wu, H., Feng, W., Chen, J., Chan, L.-N., Huang, S., and Zhang, M. (2007). PDZ Domains of Par-3 as Potential Phosphoinositide Signaling Integrators. Mol Cell 28, 886-898.

Yamashiro, S., and Watanabe, N. (2017). Overview of Single-Molecule Speckle (SiMS) Microscopy and Its Electroporation-Based Version with Efficient Labeling and Improved Spatiotemporal Resolution.

Sensors 17, 1585.

Ziemba, B.P., and Falke, J.J. (2013). Lateral diffusion of peripheral membrane proteins on supported lipid bilayers is controlled by the additive frictional drags of (1) bound lipids and (2) protein domains penetrating into the bilayer hydrocarbon core. Chem Phys Lipids 172, 67-77. 
A

D

bioRxiv preprint doi: https://doi.org/10.1101/2021.06.07.447386; this version posted June 7.2021. The copyright holder for this qreprint (which was not centifiechby? peipdeiview)

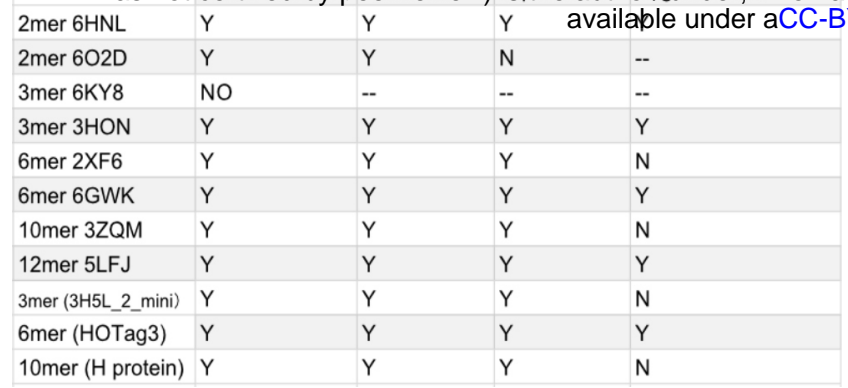

LSFGP::SNAPf

(LP566)

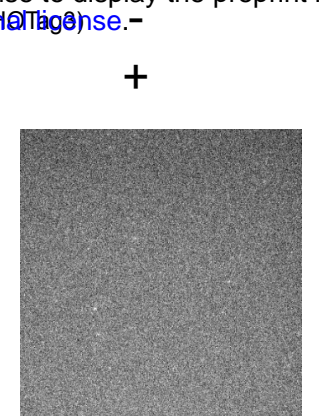

$+$

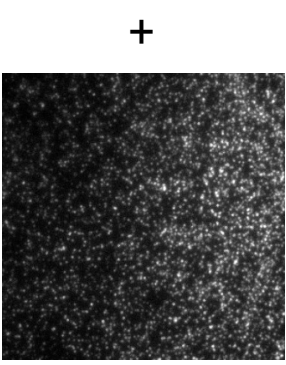

B
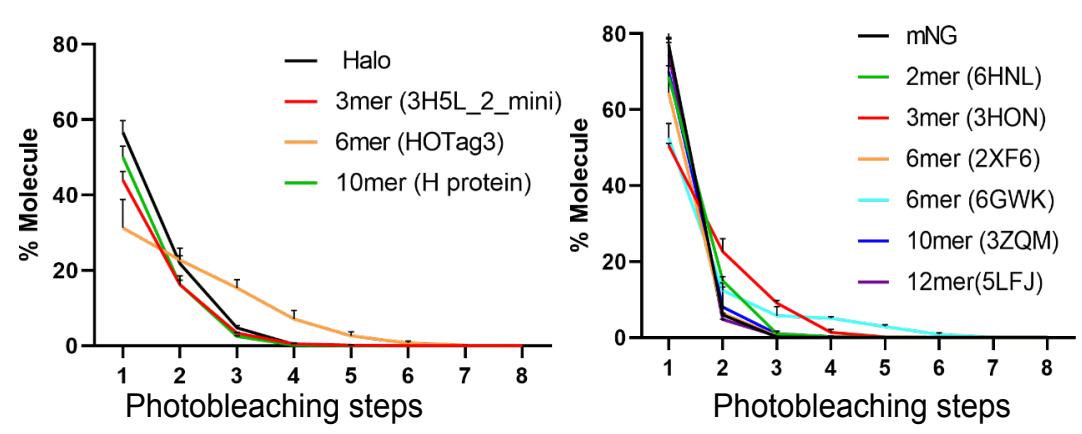

C
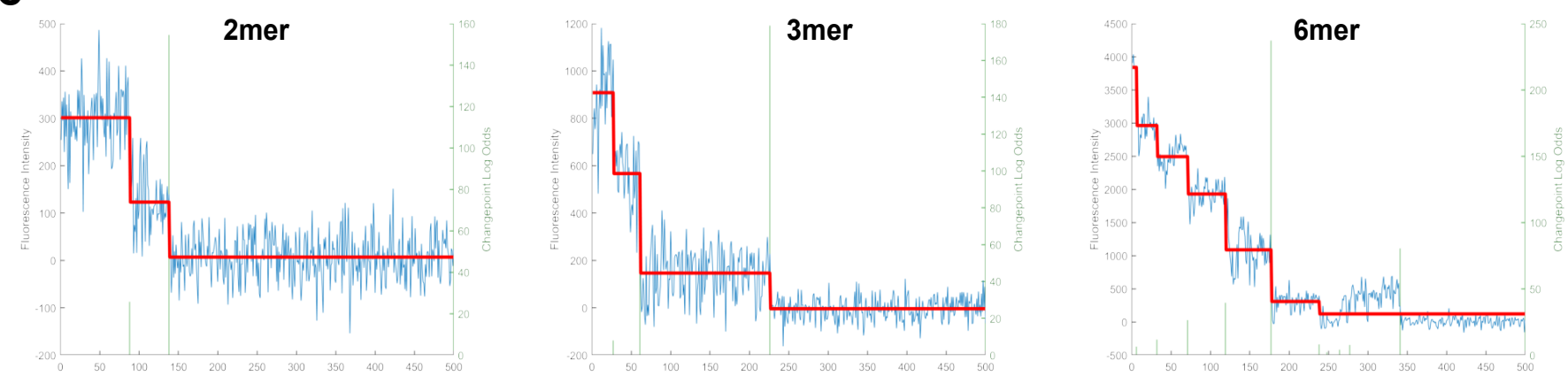

\section{E}
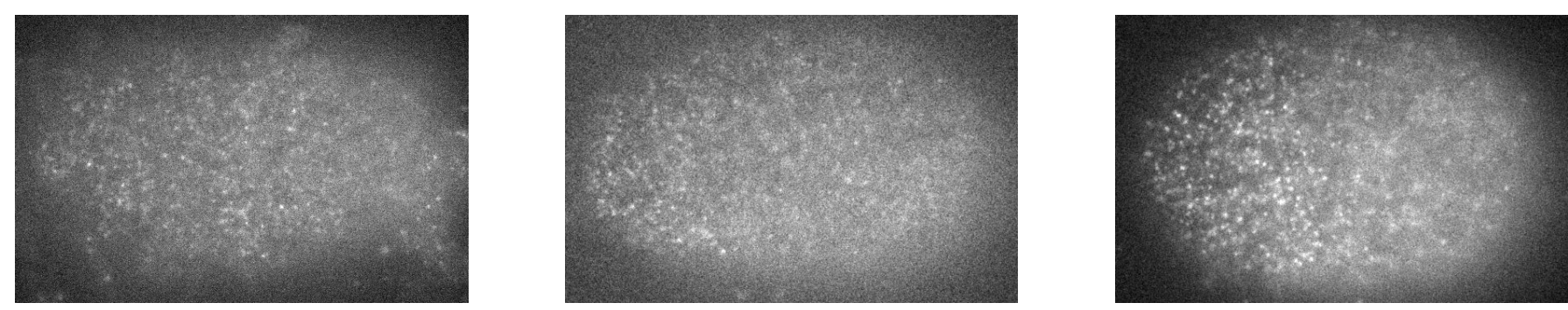

No polarization $57 \%(8 / 14)$

Weak polarization $14 \%(2 / 14)$

Strong polarization $28 \%(4 / 14)$ 


\section{Figure $\mathbf{S 1}$}

(A) Summary of the potential EOD domains tested.

(B) The validation of EOD oligomer sizes using sc-SiMpull followed by photobleaching step counting. (3 replicates for each strain). Top: EODs attached to Halo and tested by photobleaching Halo and EODs attached to $\mathrm{mNG}$ and tested by photobleaching $\mathrm{mNG}$. Please note that under this methodology, the photobleaching steps are predicted to be smaller than the real number of molecules in an oligomer due to the non-perfect maturation rate $(50-80 \%)$ of fluorescent proteins and simultaneous bleaching of multiple fluorophores.

(C) Examples of photobleaching traces of 2 mer, 3 mer and 6 mer bleaching in 2 steps, 3 steps and 6 steps respectively.

(D) sc-SiMpull assay testing the binding between GFP nanobody and GFP. The device surface was functionalized with anti-Halo antibodies. First, a GFPnb::Halo::6mer embryo was lysed to capture the EOD construct by means of the Halo - anti-Halo interaction. Then, a second embryo expressing sfGFP::SNAPf embryo was lysed to test whether the GFP nanobody, present in the EOD construct, could capture sfGFP. TIRF imaging for GFP signal.

(E) The degree of polarity defect in the dimer strain and the fraction of each category. 
A

Figure S2
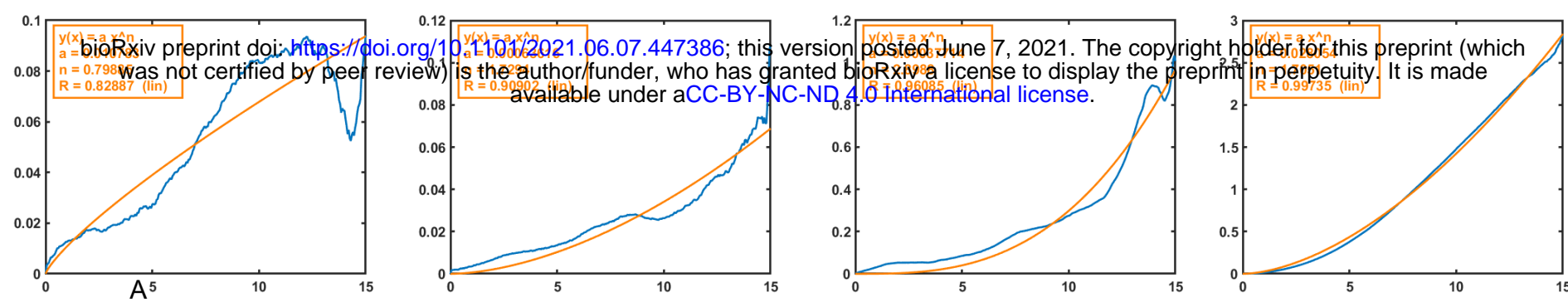

B
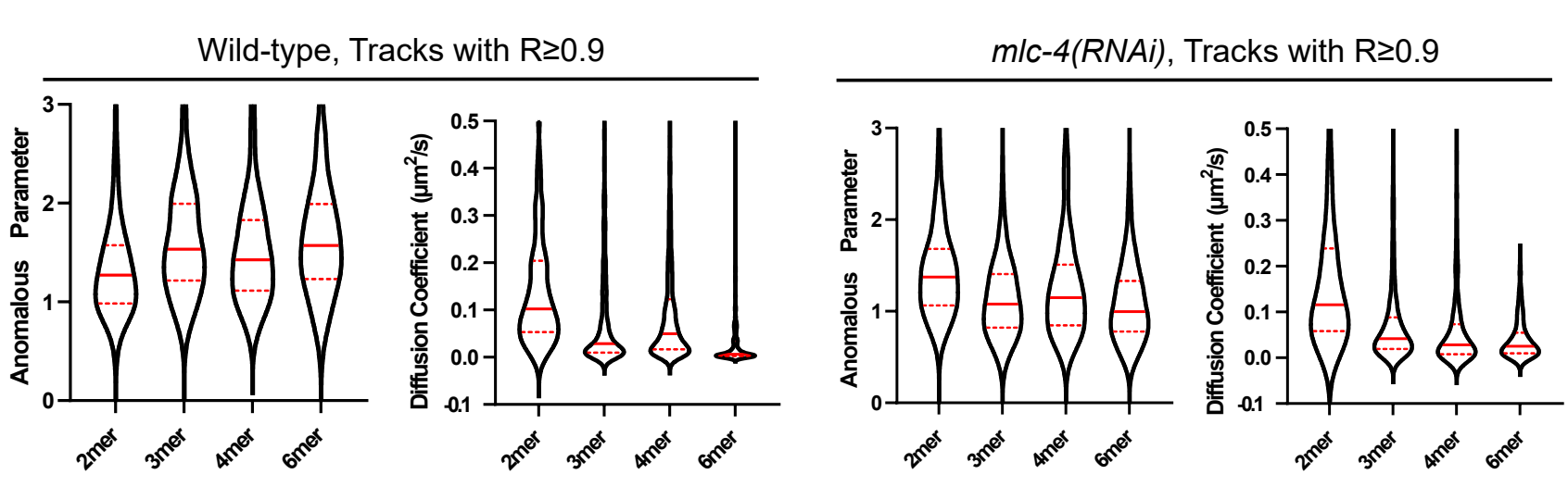

Yiran Chang \& Daniel J. Dickinson 2021, p. 35 
bioRxiv preprint doi: https://doi.org/10.1101/2021.06.07.447386; this version posted June 7, 2021. The copyright holder for this preprint (which was not certified by peer review) is the author/funder, who has granted bioRxiv a license to display the preprint in perpetuity. It is made available under aCC-BY-NC-ND 4.0 International license.

\section{Figure $\mathbf{S 2}$}

(A) Examples of a range of $\mathrm{R}$ values resulting from MSD plot curve fitting.

(B) Measured anomalous parameter and diffusion coefficient with the $\mathrm{R}$ filter set to 0.9 (an $\mathrm{R}$ value filter of 0.98 is used for the main figures). Solid lines indicate the mean. Dashed lines indicate upper and lower quartiles. Data points pooled from 3 embryos. 


\section{Figure S3}

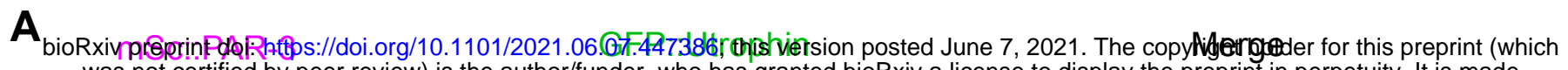
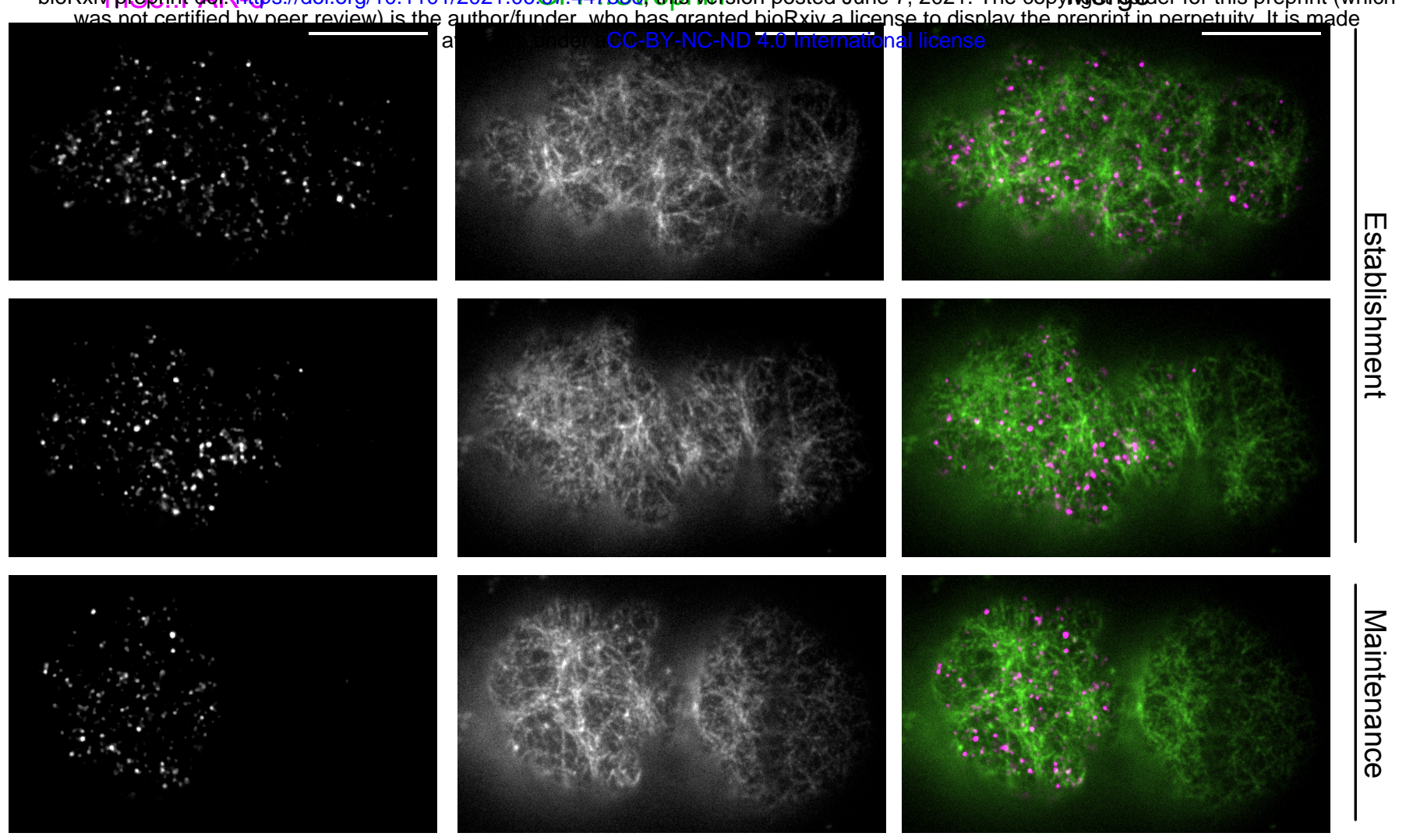

B
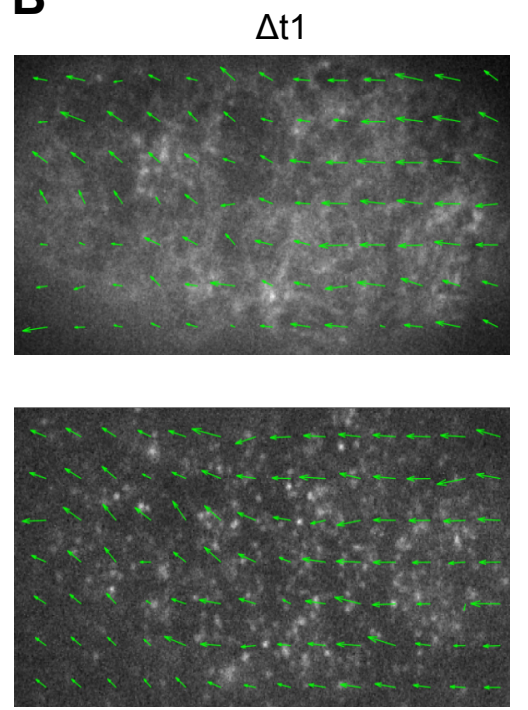

$\Delta \mathrm{t} 2$

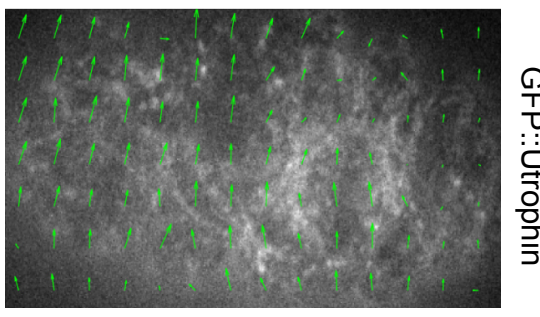

C

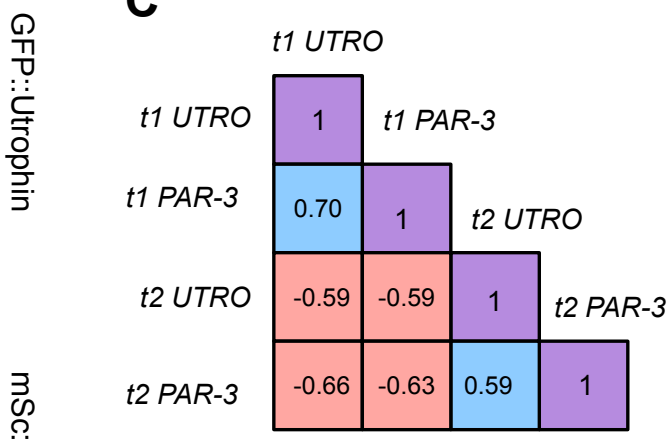


bioRxiv preprint doi: https://doi.org/10.1101/2021.06.07.447386; this version posted June 7, 2021. The copyright holder for this preprint (which was not certified by peer review) is the author/funder, who has granted bioRxiv a license to display the preprint in perpetuity. It is made available under aCC-BY-NC-ND 4.0 International license.

\section{Figure S3}

(A) TIRF imaging of mSc::PAR-3;GFP::UTRO zygote. Anterior to the left. Scale bar $=10 \mu \mathrm{m}$.

(B) The vector field generated by PIVlab at the polarization stage.

(C) Pearson's correlation of the vector fields in (B). Blue boxes represent experiments where the vector field of actin and PAR-3 from the same time point is compared, red boxes represent negative controls where unrelated images are compared, and purple boxes serve as positive controls where the same image is compared to itself. 


\section{Figure S4}

bioRxiv preprint doi: https://doi.org/10.1101/2021.06.07.447386; this version posted June 7, 2021. The copyright holder for this preprint (which

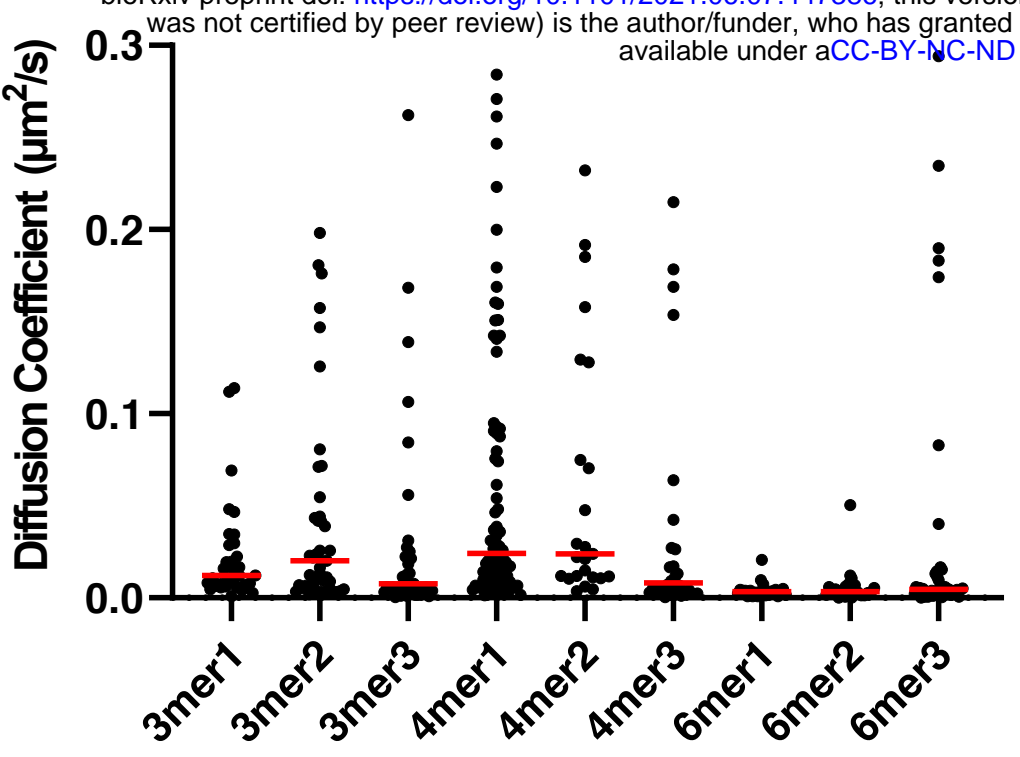


bioRxiv preprint doi: https://doi.org/10.1101/2021.06.07.447386; this version posted June 7, 2021. The copyright holder for this preprint (which was not certified by peer review) is the author/funder, who has granted bioRxiv a license to display the preprint in perpetuity. It is made available under aCC-BY-NC-ND 4.0 International license.

\section{Figure S4}

Measurements of the diffusion coefficient in individual embryos from EOD strains. Each dot corresponds to the diffusion coefficient of a single engineered PAR-3 cluster. 
A

E

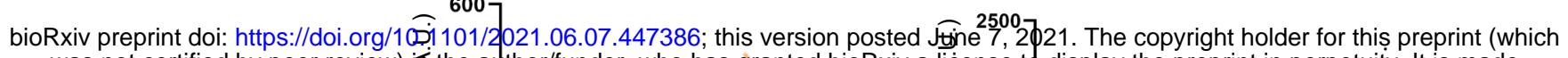
Absolute Whas not certified by peer review) 这 the aythorffunder, who has granted bioRxiv a licensegto display the preprint in perpetuity. It is made (Arbitrary Unit)

Plot in log scale

Mean calculated from the log normal distribution

All embryos normalized to log normal mean_red $=1$
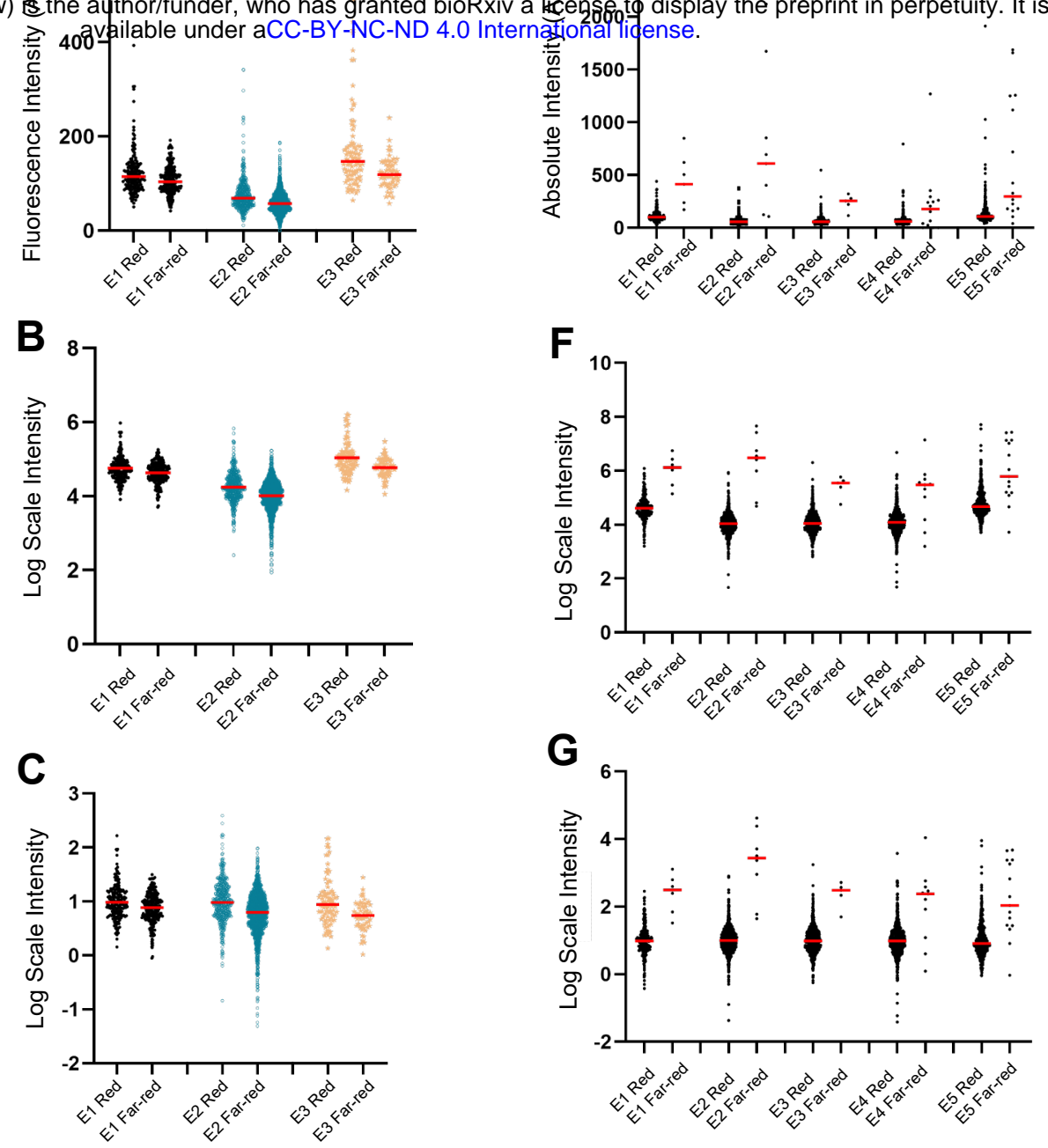

Sparse/Sparse:

Intensity/farred molecule in normalized log/normal scale calculated

Sparse/Abundant dilution: revert from log scale back to normal scale.
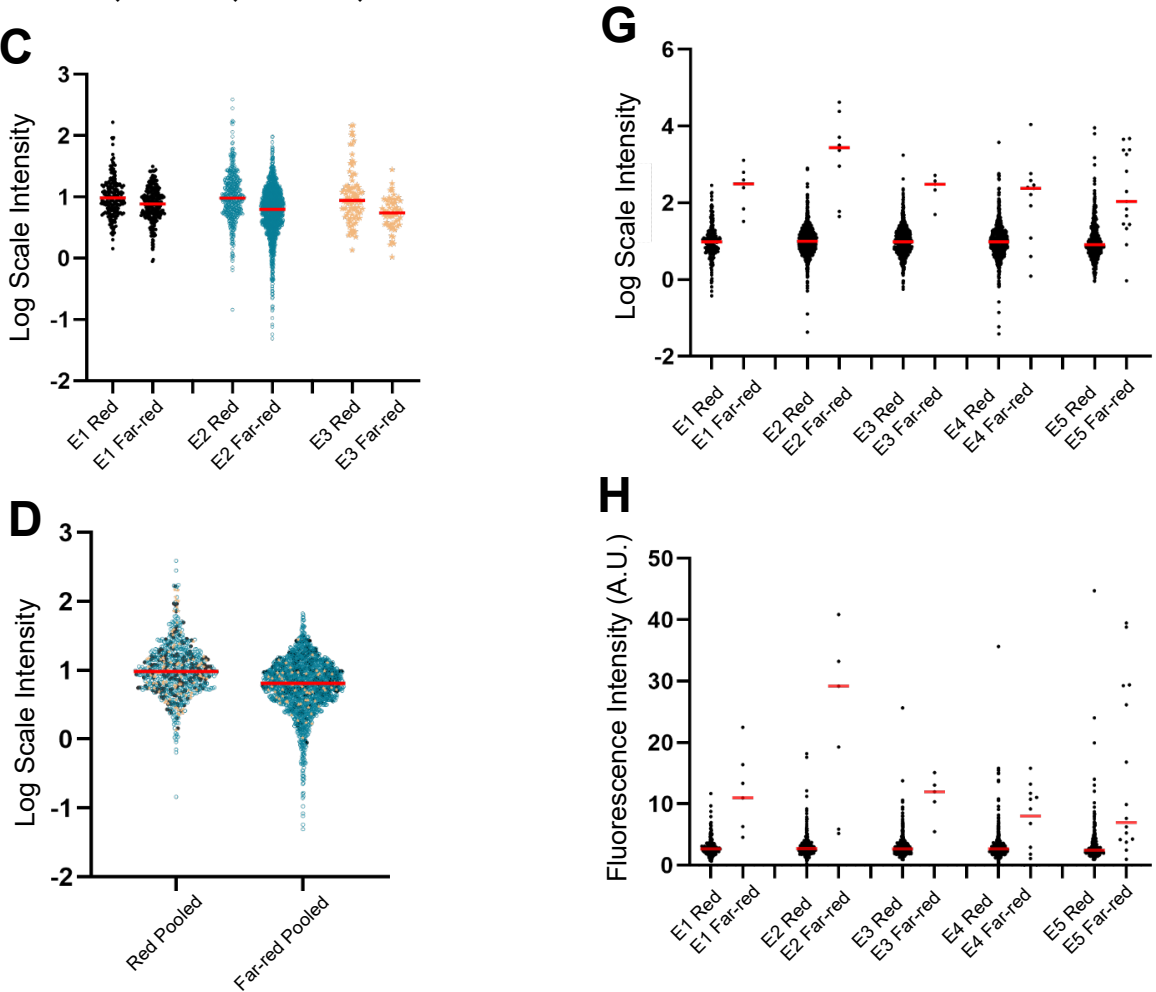

$J$

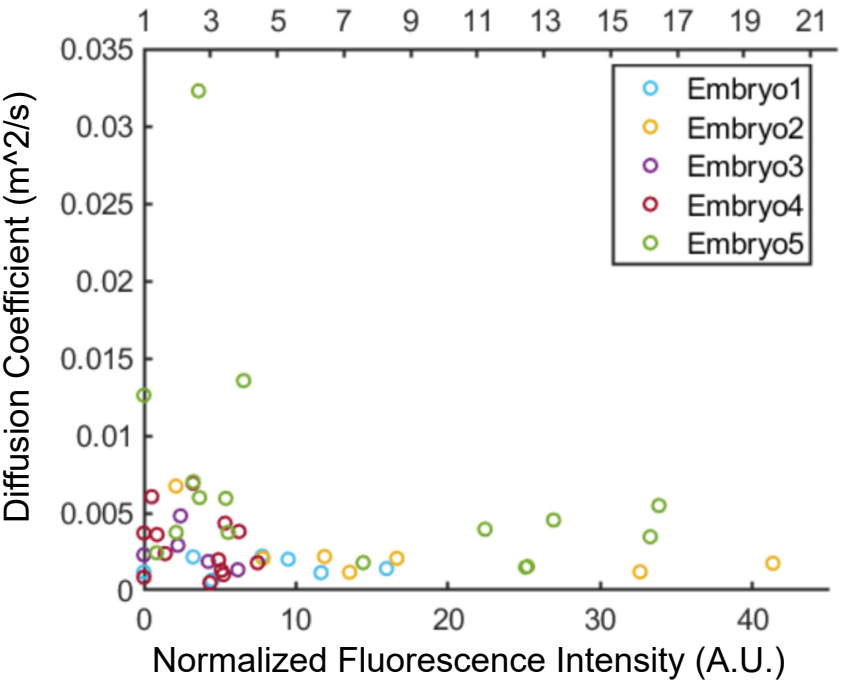

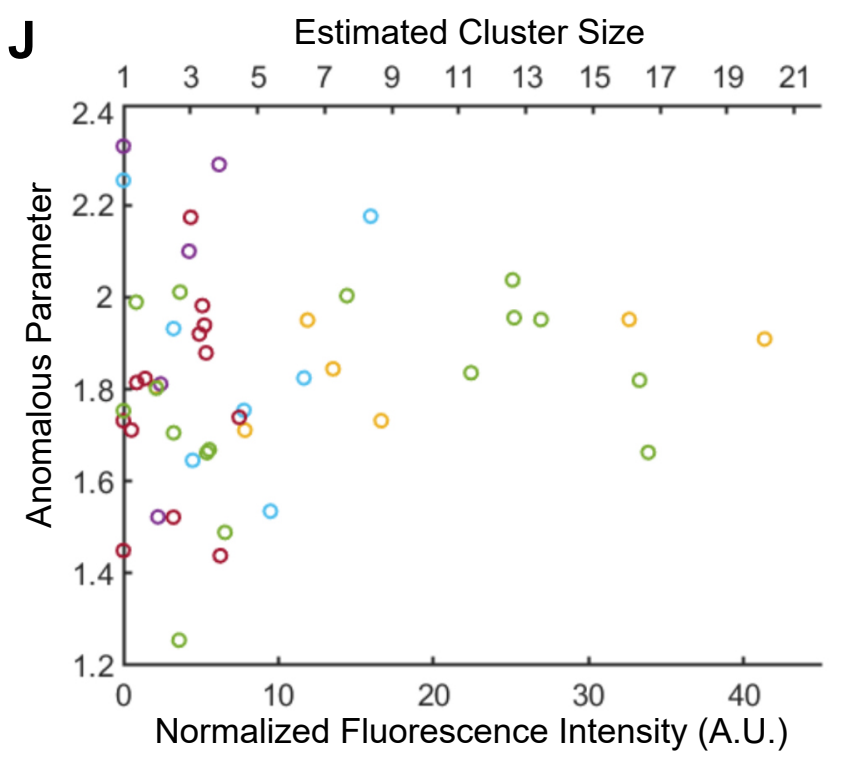




\section{Figure S5}

(A) The global intensity of red and farred signals in double-dilution experiments. Each dot represents a single foci detected. Red lines indicate the means.

(B) The log scale intensity of (A).

(C) The datasets in (B) are normalized to 1.

(D) All 3 datasets from the same color channel are pooled together for calculating the single $\mathrm{red} /$ single far-red ratio.

(E) The global intensity of red and the farred channel intensity of particles of interest in sparse/abundant dual-labeling experiments. Each dot represents a single foci detected. Red lines indicate the means.

$(F)$ The log scale intensity of (E).

$(G)$ The datasets in (F) are normalized to 1.

$(\mathrm{H})$ The normalized log scale intensity is converted back to normal scale, ready for converting into cluster size.

(I) Individual datasets presentation of anomalous parameters as a function of relative size.

$\mathrm{J}$ Individual datasets presentation of diffusion coefficient as function of relative size. 
bioRxiv preprint doi: https://doi.org/10.1101/2021.06.07.447386; this version posted June 7, 2021. The copyright holder for this preprint (which was not certified by peer review) is the author/funder, who has granted bioRxiv a license to display the preprint in perpetuity. It is made available under aCC-BY-NC-ND 4.0 International license.

\section{Movie Legends:}

Movie S1. TIRF live imaging of polarizing zygotes from strains with genetic modifications that result in different PAR-3 cluster size. Imaged at 3 seconds/frame. Anterior to the left.

Movie S2. iSIM live imaging of polarizing mNG::PAR-3; NMY-2::mKate2 zygote. Imaged at 3 seconds/frame. Anterior to the left.

Movie S3. TIRF live imaging of polarizing mSc::PAR-3; GFP::utrophin zygote. Imaged at 3 seconds/frame. Anterior to the left. 